\title{
First-Order Shape Derivative of the Energy for Elastic Plates with Rigid Inclusions and Interfacial Cracks
}

\author{
Evgeny Rudoy ${ }^{1} \cdot$ Viktor Shcherbakov $^{1,2}$ (1) \\ Accepted: 7 October 2020 / Published online: 16 November 2020 \\ (c) The Author(s) 2020
}

\begin{abstract}
Within the framework of Kirchhoff-Love plate theory, we analyze a variational model for elastic plates with rigid inclusions and interfacial cracks. The main feature of the model is a fully coupled nonpenetration condition that involves both the normal component of the longitudinal displacements and the normal derivative of the transverse deflection of the crack faces. Without making any artificial assumptions on the crack geometry and shape variation, we prove that the first-order shape derivative of the potential deformation energy is well defined and provide an explicit representation for it. The result is applied to derive the Griffith formula for the energy release rate associated with crack extension.
\end{abstract}

Keywords Kirchhoff-Love elastic plate · Rigid inclusion · Interfacial crack · Variational model $\cdot$ Shape derivative of energy $\cdot$ Griffith formula

Mathematics Subject Classification 35J87, 35Q74, 49J52, 49Q10, 74R10

\section{Introduction}

Recent and ongoing advances in engineering and material science have increased the need for mathematical tools in order to design and optimize in an efficient way three-dimensional highly inhomogeneous thin structures.

In this paper, we confine our attention to the model of a composite KirchhoffLove plate proposed in [17]. More precisely, we consider an elastic plate containing a partially debonded rigid inclusion of regular shape. The displacement field of the

\footnotetext{
$\bowtie \quad$ Viktor Shcherbakov

shcherbakov@mathematik.uni-kassel.de

Evgeny Rudoy

rem@hydro.nsc.ru

1 Lavrentyev Institute of Hydrodynamics, Lavrentyev Ave. 15, Novosibirsk, Russia 630090

2 Institute of Mathematics, University of Kassel, Heinrich-Plett-Str. 40, 34132 Kassel, Germany
} 
inclusion is specified by an equality constraint and determined completely by six unknown constants. In order to eliminate nonphysical interpenetration of the crack faces, an inequality constraint that involves both the normal component of the longitudinal displacements and the normal derivative of the transverse deflection of the crack faces is imposed. This implies a full coupling between the longitudinal displacements and transverse deflection of the plate. Our interest is in investigating the differentiability of the potential deformation energy with respect to the shape variations of the reference configuration and in finding an explicit form of the corresponding first-order shape derivative. The motivation for such a study lies in the fact that this derivative can be used to calculate efficiently the energy release rates associated with variation of defects, which are utmost of importance to predict crack propagation [4,5,12,20].

The principal difficulty in the problem at hand comes from the equality and inequality constraints, which do not preserve their structure under domain variations. To overcome this difficulty, we develop an approach that is purely based on the minimizing properties of variational solutions. The most important idea behind our technique originates from [40], where the Griffith formula for the energy release rate associated with crack extension was deduced in a rigorous way for two-dimensional elastic bodies with curvilinear cracks subjected to the Signorini conditions. Following this idea, in the case of Signorini-type constraints imposed on crack faces, general results on the shape differentiability of the potential deformation energy supported by explicit formulae for the first-order shape derivative were derived in [41] for linear elastic materials, in [32] for a Mindlin-Timoshenko elastic plate model, and in [42] for a Kirchhoff-Love elastic plate model. Another approach to the shape differentiability of the potential deformation energy based on primal-dual Lagrange formulations of the nonlinear crack problems goes back to [25,26] and the subsequent [15]. Recent developments of the primal-dual shape sensitivity analysis in smooth domains and some applications to fluid mechanics problems with divergence-free constraints were given in $[7,13,28,47]$. We finally refer to [1,24] for a derivation of the Griffith formula for elastic materials with cracks by using the Piola transform. We stress here that all of the cited works related to solid mechanics, except [42], tackle inequality constraints on the crack faces that do not involve the gradient of displacement fields, while in the model under consideration the nonpenetration condition depends on the normal derivative of the transverse deflection. This additional difficulty essentially affects the computation of the shape derivative of the potential deformation energy.

In the context of Kirchhoff-Love plate theory, the nonpenetration condition for vertical cracks was put forward in [16]. A dimension reduction from $3 \mathrm{D}$ to $2 \mathrm{D}$ in the Kirchhoff-Love energy scaling regime for rate-independent fracture processes has been the subject of research in two past decades. To our best knowledge, all of the results are restricted to the use of the notion of global energetic solutions [34]; here we just mention a couple of them. With the help of evolutionary $\Gamma$-convergence [35], a two-dimensional model for the propagation of a single crack in a plate from a threedimensional linearly elastic model involving a Barenblatt-like cohesive crack surface energy was obtained in [8]. A similar scenario for deriving two-dimensional models in the cases of Griffith-type delamination and an adhesive contact was performed in [9]. The advantages of the energetic formulation are that the initial problems and their two-dimensional counterparts have a derivative-free form and hence do not require 
any formulae for the energy release rate associated with crack extension; moreover, the crack path is not prescribed in advance.

In the presence of a preexisting interfacial crack between strongly dissimilar materials, it is natural to assume that the interface is the weakest fracture path and to apply the Griffith criterion [12] in order to describe propagation of the crack along the prescribed path. We recall that the Griffith criterion states: a crack is stationary if the energy release rate associated with crack extension is less than the fracture toughness of a material, and otherwise the crack grows. The main result of the present study gives a closed formula for the first-order shape derivative of the potential deformation energy (Theorem 2.2), and its application consists in deducing the Griffith formula for the energy release rate associated with extension of the crack along the interface, under minimal assumptions on the regularity of the crack path (Remark 2.11). Recent results in the same spirit of our work are [29,39,43,45].

Fracture behavior depends on many factors, among them the mutual dislocation and interaction of inclusions and cracks. In a series of papers $[23,30,31,44]$, this issue was treated by an optimization technique based on regular shape variations. A more delicate shape-topological analysis of the nonlinear crack problems aimed at retarding or avoiding the crack propagation process was carried out in [22,27,33,49]. Inverse problems for the nondestructive determination of elastic and rigid inclusions embedded in Kirchhoff-Love bending elastic plates were investigated in [3,36,37].

The layout of the paper is as follows. In Sect. 1, we discuss the mechanical model and present the functional framework used to assert the existence of the variational solutions. After the necessary preliminaries regarding the velocity method, which we employ to describe shape variations of the reference configuration, we formulate and prove in Sect. 2 our main result. The paper closes with a short discussion of the case when the elastic properties of the plate are inhomogeneous and an application of the result to fracture mechanics: we derive the Griffith formula for the energy release rate associated with crack extension.

\section{Formulation of the Problem}

We consider a composite plate, which is a planar thin-walled structure, within the confines of linear Kirchhoff-Love theory. The 3D undamaged plate in the reference configuration is the right cylinder

$$
\Omega^{3 \mathrm{D}}=\left\{\left(x_{1}, x_{2}, x_{3}\right) \in \mathbb{R}^{3}: x=\left(x_{1}, x_{2}\right) \in \Omega, x_{3} \in(-h, h)\right\},
$$

where $\Omega \subset \mathbb{R}^{2}$ defines the midsurface of the plate and $2 h$ denotes the thickness, which we assume to be constant. The midsurface $\Omega$ is a bounded simply connected domain with boundary $\partial \Omega$ of class $C^{1,1}$, and let $\omega_{0}$ be a compact simply connected subdomain of $\Omega$ (i.e., $\omega_{0} \Subset \Omega$ ), with boundary $\partial \omega_{0}$ of class $C^{1,1}$. The boundary $\partial \omega_{0}$ is the union of two disjoint simple curves $\gamma_{0}$ and $\partial \omega_{0} \backslash \gamma_{0}$, where $\gamma_{0}$ is a relatively open set. The outward pointing unit normal to $\partial \omega_{0}$ as well as to $\partial \Omega$ is denoted by $\nu_{0}=\left(\nu_{01}, \nu_{02}\right)^{\mathrm{T}}$. Figure 1 provides a schematic visualization of the setup. 
Fig. 1 Schematic of the midsurface of the plate

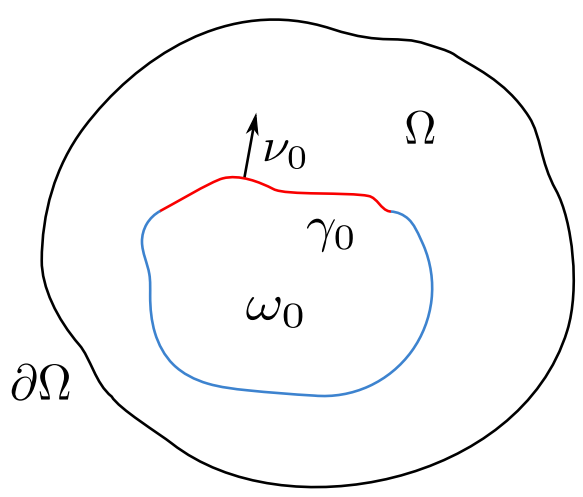

We make also the following assumption on the geometry of the non-Lipschitz domain $\Omega_{0}=\Omega \backslash \bar{\gamma}_{0}$ :

G1 There exist two domains $\Omega_{1}$ and $\Omega_{2}$ with Lipschitz boundaries such that $\bar{\Omega}_{1} \cup$ $\bar{\Omega}_{2}=\bar{\Omega}, \gamma_{0} \subset \partial \Omega_{1} \cap \partial \Omega_{2}$, and $\mathcal{H}^{1}\left(\partial \Omega_{i} \cap \partial \Omega\right)>0, i=1,2$, where $\mathcal{H}^{1}$ stands for the one-dimensional Hausdorff measure.

The region $\left(\Omega \backslash \bar{\omega}_{0}\right)^{3 \mathrm{D}}$ is occupied by a linearly elastic homogeneous material whose properties are characterized by a fourth-order tensor $\mathbb{C}^{3 \mathrm{D}}$ that is supposed to be positive, that is, there exists $c_{\mathbb{C}^{3 D}}>0$ such that

$$
\mathbb{C}^{3 \mathrm{D}} X: X \geq c_{\mathbb{C}^{3 \mathrm{D}}}|X|^{2} \text { for all } X \in \mathbb{R}_{\text {sym }}^{3 \times 3},
$$

symmetric

$$
\mathbb{C}_{i j k l}^{3 \mathrm{D}}=\mathbb{C}_{i j l k}^{3 \mathrm{D}}=\mathbb{C}_{k l i j}^{3 \mathrm{D}}, \quad i, j, k, l=1,2,3,
$$

and monoclinic symmetric with respect to the $\left(x_{1}, x_{2}\right)$-plane, which implies

$$
\mathbb{C}_{i j k 3}^{3 \mathrm{D}}=\mathbb{C}_{i 333}^{3 \mathrm{D}}=0, \quad i, j, k=1,2 .
$$

We refer the reader to Remark 2.10 for some comments on the choice of the elasticity tensor. The region $\omega_{0}^{3 \mathrm{D}}$ corresponds to a rigid inclusion, while the cylindrical surface $\gamma_{0}^{3 \mathrm{D}}$ is an interfacial crack. As usual in the theory of thin-walled structures, we deal with quantities that refer to the midsurface of the undeformed plate $\Omega_{0}$. Let $W=\left(w_{1}, w_{2}\right)^{\mathrm{T}}$ be the longitudinal displacements of the midsurface points in the $\left(x_{1}, x_{2}\right)$-plane, and $w$ is the vertical deflection of the midsurface points along the $x_{3}$-axis. In what follows, we use a variational approach to equilibrium and thus look for $(W, w)$ belonging to the Cartesian product of Sobolev spaces $H^{1}\left(\Omega_{0}\right)^{2} \times H^{2}\left(\Omega_{0}\right)$.

Let $\mathbb{C}$ be the fourth-order tensor with components

$$
\mathbb{C}_{i j k l}=\mathbb{C}_{i j k l}^{3 \mathrm{D}}-\frac{\mathbb{C}_{i j 33}^{3 \mathrm{D}} \mathbb{C}_{k l 33}^{3 \mathrm{D}}}{\mathbb{C}_{3333}^{3 \mathrm{D}}}, \quad i, j, k, l=1,2
$$


Hence the resultant membrane stress tensor $\sigma$ can be expressed in terms of the membrane strain tensor $\varepsilon(W)=1 / 2\left(\nabla_{x} W+\left(\nabla_{x} W\right)^{\mathrm{T}}\right)$ via

$$
\sigma=2 h \mathbb{C} \varepsilon(W),
$$

and the bending moment tensor $m$ is related to the bending strain tensor $-\nabla_{x}^{2} w$ through the constitutive equations

$$
m=-\frac{2 h^{3}}{3} \mathbb{C} \nabla_{x}^{2} w .
$$

The restriction of a displacement field $(W, w)$ on the domain $\omega_{0}$ is an element of the Cartesian product $R_{p}\left(\omega_{0}\right) \times R_{v}\left(\omega_{0}\right)$, where $R_{p}\left(\omega_{0}\right)$ and $R_{v}\left(\omega_{0}\right)$ are the spaces of infinitesimal rigid displacement fields on $\omega_{0}$ that represent ker $\varepsilon$ and $\operatorname{ker} \nabla_{x}^{2}$, respectively:

$$
\begin{aligned}
& R_{p}\left(\omega_{0}\right)=\left\{W \in H^{1}\left(\omega_{0}\right)^{2}: \varepsilon(W)=0 \text { in } \omega_{0}\right\}, \\
& R_{v}\left(\omega_{0}\right)=\left\{w \in H^{2}\left(\omega_{0}\right): \nabla_{x}^{2} w=0 \text { in } \omega_{0}\right\} .
\end{aligned}
$$

We observe that the following identities hold true [48, Lemma 1.1; Chapter III, Subsection 2.1.1]

$$
\begin{aligned}
& R_{p}\left(\omega_{0}\right)=\left\{W(x)=B \operatorname{Id}_{x}(x)+C: B \in \mathbb{R}^{2 \times 2}, B=-B^{\mathrm{T}}, C \in \mathbb{R}^{2}, x \in \omega_{0}\right\}, \\
& R_{v}\left(\omega_{0}\right)=\left\{w(x)=A \cdot \operatorname{Id}_{x}(x)+a: A \in \mathbb{R}^{2}, a \in \mathbb{R}, x \in \omega_{0}\right\} .
\end{aligned}
$$

The regularity assumptions on the domains $\omega_{0}$ and $\Omega \backslash \bar{\omega}_{0}$ provide that the trace operators

$$
\begin{aligned}
& \operatorname{Tr}_{k, \omega_{0}}: H^{k}\left(\omega_{0}\right)^{k_{*}} \rightarrow \prod_{i=0}^{k-1} H^{k-i-1 / 2}\left(\partial \omega_{0}\right)^{k_{*}}, \\
& \operatorname{Tr}_{k, \Omega \backslash \bar{\omega}_{0}}: H^{k}\left(\Omega \backslash \bar{\omega}_{0}\right)^{k_{*}} \rightarrow \prod_{i=0}^{k-1} H^{k-i-1 / 2}\left(\partial \omega_{0} ; \partial \Omega\right)^{k_{*}},
\end{aligned}
$$

where the superscript $k_{*}$ is equal to 2 if $k=1$ and 1 if $k=2$, are well defined and continuous. Moreover, these operators have right continuous inverses (lifting operators) [14, Theorem 1.5.1.2], which we denote by $\operatorname{Tr}_{k, \omega_{0}}^{-1}$ and $\operatorname{Tr}_{k, \Omega \backslash \bar{\omega}_{0}}^{-1}$, respectively. According to the positive and negative directions of the unit normal $\nu_{0}$ on $\partial \omega_{0}$, there is a positive face $\partial \omega_{0}^{+}$and a negative face $\partial \omega_{0}^{-}$. The double brackets $\llbracket v \rrbracket=v^{+}-v^{-}$ stand for the jump of a function $v$ across $\partial \omega_{0}$. For convenience, the same notation serves as well for the jump of functions across another smooth curves, it always clear from the context. In order to eliminate interpenetration of the crack faces, we impose the inequality restriction

$$
\llbracket W \rrbracket \cdot v_{0} \geq h\left|\llbracket \nabla_{x} w \rrbracket \cdot v_{0}\right| \quad \text { on } \quad \gamma_{0},
$$


which is understood in the sense of traces. We emphasize that, from a mechanical point of view, the nonpenetration condition (4) describes the interaction of the opposite crack faces, admitting their frictionless contact (the equality case) or the absence of contact (the inequality case), the contact surface being unknown a priori.

We finally suppose that the plate is clamped along its outer edge, which leads to the homogeneous boundary conditions

$$
W=(0,0)^{\mathrm{T}}, \quad w=\nabla_{x} w \cdot v_{0}=0 \quad \text { on } \quad \partial \Omega .
$$

The potential deformation energy of the system associated with a displacement field $(W, w)$ is the stored elastic energy minus the work of external loadings $(F, f) \in$ $C^{1}(\bar{\Omega})^{2} \times C^{1}(\bar{\Omega})$ :

$$
\begin{aligned}
\mathcal{E}\left(\Omega_{0} ; W, w\right)= & \frac{1}{2} \int_{\Omega \backslash \bar{\omega}_{0}} \sigma(W): \varepsilon(W) \mathrm{d} x-\frac{1}{2} \int_{\Omega \backslash \bar{\omega}_{0}} m(w): \nabla_{x}^{2} w \mathrm{~d} x \\
& -\int_{\Omega_{0}} F \cdot W \mathrm{~d} x-\int_{\Omega_{0}} f w \mathrm{~d} x .
\end{aligned}
$$

The variational principle of minimum potential deformation energy forces that the composite plate $\Omega_{0}^{3 \mathrm{D}}$ clamped along the outer edge, with elastic part $\left(\Omega \backslash \bar{\omega}_{0}\right)^{3 \mathrm{D}}$, rigid part $\omega_{0}^{3 \mathrm{D}}$, interfacial crack $\gamma_{0}^{3 \mathrm{D}}$, and potential deformation energy given by (5), is in equilibrium if the displacement field of its midsurface $\left(W_{0}, w_{0}\right)$ is a solution of the minimization problem

$$
\inf _{(W, w) \in K_{0}\left(\Omega_{0}\right)} \mathcal{E}\left(\Omega_{0} ; W, w\right),
$$

where the set of kinematically admissible displacement fields reads as follows

$$
\begin{gathered}
K_{0}\left(\Omega_{0}\right)=\left\{(W, w) \in H_{\partial \Omega}^{1}\left(\Omega_{0}\right)^{2} \times H_{\partial \Omega}^{2}\left(\Omega_{0}\right):\left.(W, w)\right|_{\omega_{0}} \in R_{p}\left(\omega_{0}\right) \times R_{v}\left(\omega_{0}\right)\right. \\
\left.\llbracket W \rrbracket \cdot v_{0} \geq h\left|\llbracket \nabla_{x} w \rrbracket \cdot v_{0}\right| \text { on } \gamma_{0}\right\}
\end{gathered}
$$

The convex set $K_{0}\left(\Omega_{0}\right)$ is closed in the Cartesian product $H_{\partial \Omega}^{1}\left(\Omega_{0}\right)^{2} \times H_{\partial \Omega}^{2}\left(\Omega_{0}\right)$ of the Sobolev spaces

$$
\begin{aligned}
& H_{\partial \Omega}^{1}\left(\Omega_{0}\right)=\left\{v \in H^{1}\left(\Omega_{0}\right): v=0 \text { on } \partial \Omega\right\}, \\
& H_{\partial \Omega}^{2}\left(\Omega_{0}\right)=\left\{v \in H^{2}\left(\Omega_{0}\right): v=\nabla_{x} v \cdot v_{0}=0 \text { on } \partial \Omega\right\} .
\end{aligned}
$$

Since $\mathcal{E}$ is convex and Gâteux-differentiable, the minimization problem (6) is equivalent to the variational inequality

$$
\left(W_{0}, w_{0}\right) \in K_{0}\left(\Omega_{0}\right), \int_{\Omega \backslash \bar{\omega}_{0}} \sigma\left(W_{0}\right): \varepsilon\left(\bar{W}-W_{0}\right) \mathrm{d} x-\int_{\Omega \backslash \bar{\omega}_{0}} m\left(w_{0}\right): \nabla_{x}^{2}\left(\bar{w}-w_{0}\right) \mathrm{d} x
$$




$$
-\int_{\Omega_{0}} F \cdot\left(\bar{W}-W_{0}\right) \mathrm{d} x-\int_{\Omega_{0}} f\left(\bar{w}-w_{0}\right) \mathrm{d} x \geq 0 \quad \forall(\bar{W}, \bar{w}) \in K_{0}\left(\Omega_{0}\right) .
$$

Thanks to G1, the Korn inequality and the Friedrichs inequality are valid in $\Omega_{i}$, $i=1,2$, which implies the coercivity of $\mathcal{E}$. Moreover, the energy functional $\mathcal{E}$ is weakly lower semicontinuous. The minimization problem (6) (and hence the variational inequality (7)) therefore admits a solution $\left(W_{0}, w_{0}\right) \in K_{0}\left(\Omega_{0}\right)$. It is straightforward to check that the solution to (7) is unique.

We are now ready to give the classical formulation of the equilibrium problem. We seek $\left(W_{0}, w_{0}\right)$ in the domain $\Omega_{0},\left(W_{0}^{R}, w_{0}^{R}\right) \in R_{p}\left(\omega_{0}\right) \times R_{v}\left(\omega_{0}\right)$, and $(\sigma, m)$ in the domain $\Omega \backslash \bar{\omega}_{0}$ satysfying the following equations and boundary conditions

$$
\begin{aligned}
& -\operatorname{div}_{x} \sigma=F \text { in } \Omega \backslash \bar{\omega}_{0}, \\
& \sigma-2 h \mathbb{C} \varepsilon\left(W_{0}\right)=0 \quad \text { in } \Omega \backslash \bar{\omega}_{0}, \\
& -\operatorname{div}_{x} \operatorname{div}_{x} m=f \quad \text { in } \Omega \backslash \bar{\omega}_{0}, \\
& m+\frac{2 h^{3}}{3} \mathbb{C} \nabla_{x}^{2} w_{0}=0 \text { in } \Omega \backslash \bar{\omega}_{0}, \\
& W_{0}=(0,0)^{\mathrm{T}}, \quad w_{0}=\nabla_{x} w_{0} \cdot v_{0}=0 \quad \text { on } \partial \Omega, \\
& \llbracket W_{0} \rrbracket \cdot v_{0} \geq h\left|\llbracket \nabla_{x} w_{0} \rrbracket \cdot v_{0}\right| \quad \text { on } \gamma_{0} \text {, } \\
& \sigma_{\tau_{0}}^{+}=(0,0)^{\mathrm{T}}, \quad t_{v_{0}}^{+}=0, \quad h^{-1}\left|m_{v_{0}}^{+}\right| \leq-\sigma_{\nu_{0}}^{+} \quad \text { on } \quad \gamma_{0}, \\
& \sigma_{\nu_{0}}^{+} \llbracket W_{0} \rrbracket \cdot v_{0}+m_{\nu_{0}}^{+} \llbracket \nabla_{x} w_{0} \rrbracket \cdot v_{0}=0 \text { on } \gamma_{0} \text {, } \\
& \llbracket W_{0} \rrbracket=(0,0)^{\mathrm{T}}, \quad \llbracket w_{0} \rrbracket=\llbracket \nabla_{x} w_{0} \rrbracket \cdot v_{0}=0 \quad \text { on } \quad \partial \omega_{0} \backslash \gamma_{0}, \\
& W_{0}=W_{0}^{R}, \quad w_{0}=w_{0}^{R} \text { in } \omega_{0} \text {, } \\
& -\int_{\partial \omega_{0}} \sigma^{+} \nu_{0} \cdot \bar{W}^{R} \mathrm{~d} \mathcal{H}^{1}=\int_{\omega_{0}} F \cdot \bar{W}^{R} \mathrm{~d} x \quad \forall \bar{W}^{R} \in R_{p}\left(\omega_{0}\right) \\
& \int_{\partial \omega_{0}} t_{\nu_{0}}^{+} \bar{w}^{R} \mathrm{~d} \mathcal{H}^{1}-\int_{\partial \omega_{0}} m_{v_{0}}^{+} \nabla_{x} \bar{w}^{R} \cdot v_{0} \mathrm{~d} \mathcal{H}^{1}=\int_{\omega_{0}} f \bar{w}^{R} \mathrm{~d} x \quad \forall \bar{w}^{R} \in R_{v}\left(\omega_{0}\right) .
\end{aligned}
$$

Here the traction $\sigma^{+} \nu_{0}$, transverse force $t_{\nu_{0}}^{+}$, and bending moment $m_{\nu_{0}}^{+}$acting on $\partial \omega_{0}$ are

$$
\begin{aligned}
\sigma^{+} \nu_{0} & =\left(\sigma_{1 j}^{+} v_{0 j}, \sigma_{2 j}^{+} v_{0 j}\right)^{\mathrm{T}}, \quad t_{\nu_{0}}^{+}=-\left(\operatorname{div}_{x} m\right)^{+} \cdot v_{0}-\partial_{\tau_{0}}\left(\left(m^{+} v_{0}\right) \cdot \tau_{0}\right) \\
m_{v_{0}}^{+} & =-\left(m^{+} \nu_{0}\right) \cdot v_{0}, \quad \tau_{0}=\left(\tau_{01}, \tau_{02}\right)^{\mathrm{T}}=\left(-v_{02}, v_{01}\right)^{\mathrm{T}}
\end{aligned}
$$

and the traction $\sigma^{+} \nu_{0}$ is decomposed into normal and tangential components given by

$$
\sigma_{\nu_{0}}^{+}=\sigma^{+} \nu_{0} \cdot v_{0}, \quad \sigma_{\tau_{0}}^{+}=\sigma^{+} \nu_{0}-\sigma_{\nu_{0}}^{+} \nu_{0}
$$

A repeated Roman subscript signifies summation from 1 to 2. Equations (8) and (10) are equilibrium equations for the elastic part of the plate with respect to the $\left(x_{1}, x_{2}\right)$-plane 
and $x_{3}$-axis, respectively. Conditions (13)-(15) are the complementarity conditions for contact of the crack faces. The nonlocal boundary conditions (18) and (19) ensure that the rigid part of the plate in equilibrium, that is, the resultant force and moment acting on it vanish.

It is a routine matter to verify that our minimizer $\left(W_{0}, w_{0}\right) \in K_{0}\left(\Omega_{0}\right)$ is indeed a weak solution of the Euler-Lagrange system (8)-(19), see [17].

Remark 1.1 We elucidate briefly in what sense the natural boundary conditions (14)(19) for the minimizer $\left(W_{0}, w_{0}\right)$ are satisfied. For each $k=1,2$, we introduce the Lions-Magenes space

$$
H_{00}^{k-1 / 2}\left(\gamma_{0}\right)=\left\{v \in H_{0}^{k-1 / 2}\left(\gamma_{0}\right): d^{-1 / 2} \partial_{\tau_{0}}^{i} v \in L^{2}\left(\gamma_{0}\right), 0 \leq i \leq k-1\right\},
$$

where $\partial_{\tau_{0}}^{0} v=v, \partial_{\tau_{0}}^{1} v=\partial_{\tau_{0}} v$, and $d(s)=\operatorname{dist}_{\partial \omega_{0}}\left(s, \partial \gamma_{0}\right)$. This space equipped with the norm

$$
\|v\|_{H_{00}^{k-1 / 2}\left(\gamma_{0}\right)}=\left(\|v\|_{H^{k-1 / 2}\left(\gamma_{0}\right)}^{2}+\sum_{i=0}^{k-1}\left\|d^{-1 / 2} \partial_{\tau_{0}}^{i} v\right\|_{L^{2}\left(\gamma_{0}\right)}^{2}\right)^{\frac{1}{2}}
$$

is a Banach space. Since $\partial \omega_{0} \in C^{1,1}$, the following equivalence holds [18, Proposition 1.1]

$$
v \in H_{00}^{k-1 / 2}\left(\gamma_{0}\right) \Leftrightarrow \bar{v}=\left\{\begin{array}{lll}
v & \text { on } & \gamma_{0} \\
0 & \text { on } & \partial \omega_{0} \backslash \gamma_{0}
\end{array} \quad \in H^{k-1 / 2}\left(\partial \omega_{0}\right) .\right.
$$

Denote by $\langle\cdot, \cdot\rangle_{k-1 / 2, \gamma_{0}}^{00}$ the duality pairing between the space $H_{00}^{k-1 / 2}\left(\gamma_{0}\right)$ and its topological dual space $H_{00}^{-k+1 / 2}\left(\gamma_{0}\right)$. If $\sigma, m \in L^{2}\left(\Omega \backslash \bar{\omega}_{0}\right)^{2 \times 2}, \operatorname{div}_{x} \sigma \in L^{2}\left(\Omega \backslash \bar{\omega}_{0}\right)^{2}$, and $\operatorname{div}_{x} \operatorname{div}_{x} m \in L^{2}\left(\Omega \backslash \bar{\omega}_{0}\right)$, then

$$
\sigma_{\tau_{0}}^{+} \in H_{00}^{-1 / 2}\left(\gamma_{0}\right)^{2}, \quad \sigma_{\nu_{0}}^{+}, m_{\nu_{0}}^{+} \in H_{00}^{-1 / 2}\left(\gamma_{0}\right), \quad t_{\nu_{0}}^{+} \in H_{00}^{-3 / 2}\left(\gamma_{0}\right),
$$

and the boundary conditions (14) and (15) read as follows [17]

$$
\begin{aligned}
& \left\langle\sigma_{\tau_{0}}^{+},\left.v\right|_{1 / 2, \gamma_{0}} ^{00}=0 \quad \forall v \in H_{00}^{1 / 2}\left(\gamma_{0}\right)^{2}, \quad v \cdot v_{0}=0,\right. \\
& \quad\left\langle t_{v_{0}}^{+},\left.v\right|_{3 / 2, \gamma_{0}} ^{00}=0 \quad \forall v \in H_{00}^{3 / 2}\left(\gamma_{0}\right),\right. \\
& \left\langle\sigma_{v_{0}}^{+} \pm h^{-1} m_{\nu_{0}}^{+}, v\right\rangle_{1 / 2, \gamma_{0}}^{00} \leq 0 \quad \forall v \in H_{00}^{1 / 2}\left(\gamma_{0}\right), \quad v \geq 0, \\
& \left\langle\sigma_{\nu_{0}}^{+}, \llbracket W_{0} \rrbracket \cdot v_{0}\right\rangle_{1 / 2, \gamma_{0}}^{00}+\left\langle m_{\nu_{0}}^{+},\left.\llbracket \nabla_{x} w_{0} \rrbracket \cdot v_{0}\right|_{1 / 2, \gamma_{0}} ^{00}=0,\right.
\end{aligned}
$$


whereas the interpretation of the nonlocal conditions (18) and (19) is provided by

$$
\begin{aligned}
& \left\langle\sigma^{+} \nu_{0}, v\right\rangle_{1 / 2, \partial \omega_{0}}=-\int_{\omega_{0}} F \cdot v \mathrm{~d} x \quad \forall v \in R_{p}\left(\omega_{0}\right), \\
& \left\langle t_{\nu_{0}}^{+}, v\right\rangle_{3 / 2, \partial \omega_{0}}-\left\langle m_{\nu_{0}}^{+}, \nabla_{x} v \cdot v_{0}\right\rangle_{1 / 2, \partial \omega_{0}}=\int_{\omega_{0}} f v \mathrm{~d} x \quad \forall v \in R_{v}\left(\omega_{0}\right) .
\end{aligned}
$$

Here $\langle\cdot, \cdot\rangle_{k-1 / 2, \partial \omega_{0}}$ is the duality pairing between the spaces $H^{k-1 / 2}\left(\partial \omega_{0}\right)$ and $H^{-k+1 / 2}\left(\partial \omega_{0}\right)$.

We exploit some of these conditions to derive an explicit formula for the first-order shape derivative of the potential deformation energy, see Theorem 2.2.

\section{Shape Derivative of the Energy}

We begin this section by recalling the velocity (speed) method [46, Section 2.9], which we employ to describe shape variations of the reference domain $\Omega_{0}$. Let $V \in$ $W_{\text {loc }}^{2, \infty}\left(\mathbb{R}^{2}\right)^{2}$ be a given shape velocity field. We assume further that $V$ is compactly supported in the domain $\Omega$. This assumption does not limit the generality of our analysis, but it simplifies the presentation. The following proposition is a core of the velocity method and allows us to construct a family of diffeomorphisms of $\mathbb{R}^{2}$ generated by $V$.

Proposition 2.1 Let $V \in W_{\text {loc }}^{2, \infty}\left(\mathbb{R}^{2}\right)^{2}$ be a given shape velocity field satisfying the property $\operatorname{supp} V \Subset \Omega$. Then there exists $\delta_{0}>0$ such that:

(a) the Cauchy problem

$$
\frac{d}{d \delta} T_{\delta}=V \circ T_{\delta} \text { for } \delta \neq 0, \quad T_{0}=\mathrm{Id}_{x},
$$

has a unique solution $T_{[\cdot]} \in C^{1}\left(\left(-\delta_{0}, \delta_{0}\right) ; W_{\mathrm{loc}}^{2, \infty}\left(\mathbb{R}^{2}\right)^{2}\right)$;

(b) the Cauchy problem

$$
\frac{d}{d \delta} T_{\delta}^{-1}=-V \circ T_{\delta} \text { for } \delta \neq 0, \quad T_{0}^{-1}=\mathrm{Id}_{y}
$$

has a unique solution $T_{[\cdot]}^{-1} \in C^{1}\left(\left(-\delta_{0}, \delta_{0}\right) ; W_{\text {loc }}^{2, \infty}\left(\mathbb{R}^{2}\right)^{2}\right)$;

(c) fixed $\delta \in\left(-\delta_{0}, \delta_{0}\right)$, the inverse mapping to $T_{\delta}$ is $T_{\delta}^{-1}$, that is,

$$
T_{\delta} \circ T_{\delta}^{-1}=\operatorname{Id}_{y}, \quad T_{\delta}^{-1} \circ T_{\delta}=\operatorname{Id}_{x}, \quad x, y \in \mathbb{R}^{2} .
$$

Proof The proof is carried out in [21, Section 2] for $W^{1, \infty}$ vector fields. Without any changes, the arguments are also applicable to $W^{2, \infty}$ vector fields $V$. 
Before proceeding further, we record here a consequence of [2, Theorem 2.2] that will be useful throughout the paper. For each $k=1,2$, the space $W^{k, \infty}(\Omega)$ is algebraically and topologically isomorphic to $C^{k-1,1}(\bar{\Omega})$.

Let us fix $\delta \in\left(-\delta_{0}, \delta_{0}\right)$ and set $\omega_{\delta}=T_{\delta}\left(\omega_{0}\right)$ and $\gamma_{\delta}=T_{\delta}\left(\gamma_{0}\right)$. Since $T_{\delta}$ is an orientation-preserving $C^{1,1}$-diffeomorphism between $\Omega$ and $T_{\delta}(\Omega)$, coinciding with the identity on $\partial \Omega$, we have $T_{\delta}(\Omega)=\Omega$, see [6, Theorem 5.5-2]. The domain $\omega_{\delta} \Subset \Omega$ is a simply connected one, and its boundary $\partial \omega_{\delta}=T_{\delta}\left(\partial \omega_{0}\right)$ is of class $C^{1,1}$, with the outward pointing unit normal $\nu^{\delta}$. Moreover, the boundary $\partial \omega_{\delta}$ is the union of two disjoint simple curves $\gamma_{\delta}$ and $\partial \omega_{\delta} \backslash \gamma_{\delta}$, and $\gamma_{\delta}$ is a relatively open set. So we can put $\Omega_{\delta}=\Omega \backslash \bar{\gamma}_{\delta}$. Against this background, we conclude that the mapping $T_{\delta}$ induces a $C^{1,1}$-diffeomorphism between the non-Lipschitz domains $\Omega_{0}$ and $\Omega_{\delta}$. To complete our considerations on the geometry of the perturbed domain $\Omega_{\delta}$, let us note that from the condition $\operatorname{supp} V \Subset \Omega$ follows the validity of $\mathbf{G 1}$ for $\Omega_{\delta}$.

As above, the equilibrium of the composite plate $\Omega_{\delta}^{3 \mathrm{D}}$ clamped along the outer edge, with elastic part $\left(\Omega \backslash \bar{\omega}_{\delta}\right)^{3 \mathrm{D}}$, rigid part $\omega_{\delta}^{3 \mathrm{D}}$, interfacial crack $\gamma_{\delta}^{3 \mathrm{D}}$, and potential deformation energy

$$
\begin{aligned}
\mathcal{E}\left(\Omega_{\delta} ; W, w\right)= & \frac{1}{2} \int_{\Omega \backslash \bar{\omega}_{\delta}} \sigma(W): \varepsilon(W) \mathrm{d} y \\
& -\frac{1}{2} \int_{\Omega \backslash \bar{\omega}_{\delta}} m(w): \nabla_{x}^{2} w \mathrm{~d} y-\int_{\Omega_{\delta}} F \cdot W \mathrm{~d} y-\int_{\Omega_{\delta}} f w \mathrm{~d} y,
\end{aligned}
$$

is achieved when the displacement field of its midsurface $\left(W^{\delta}, w^{\delta}\right)$ is a solution of the minimization problem

$$
\inf _{(W, w) \in K^{\delta}\left(\Omega_{\delta}\right)} \mathcal{E}\left(\Omega_{\delta} ; W, w\right)
$$

the set of kinematically admissible displacement fields being

$$
\begin{gathered}
K^{\delta}\left(\Omega_{\delta}\right)=\left\{(W, w) \in H_{\partial \Omega}^{1}\left(\Omega_{\delta}\right)^{2} \times H_{\partial \Omega}^{2}\left(\Omega_{\delta}\right):\left.(W, w)\right|_{\omega_{\delta}} \in R_{p}\left(\omega_{\delta}\right) \times R_{v}\left(\omega_{\delta}\right)\right. \\
\left.\llbracket W \rrbracket \cdot v^{\delta} \geq h\left|\llbracket \nabla_{y} w \rrbracket \cdot v^{\delta}\right| \text { on } \gamma_{\delta}\right\} .
\end{gathered}
$$

The minimization problem (20) possesses a unique solution satisfying the variational inequality

$$
\begin{aligned}
& \left(W^{\delta}, w^{\delta}\right) \in K^{\delta}\left(\Omega_{\delta}\right), \quad \int_{\Omega \backslash \bar{\omega}_{\delta}} \sigma\left(W^{\delta}\right): \varepsilon\left(\bar{W}-W^{\delta}\right) \mathrm{d} y-\int_{\Omega \backslash \bar{\omega}^{\delta}} m\left(w^{\delta}\right): \nabla_{y}^{2}\left(\bar{w}-w^{\delta}\right) \mathrm{d} y \\
& -\int_{\Omega_{\delta}} F \cdot\left(\bar{W}-W^{\delta}\right) \mathrm{d} y-\int_{\Omega_{\delta}} f\left(\bar{w}-w^{\delta}\right) \mathrm{d} y \geq 0 \quad \forall(\bar{W}, \bar{w}) \in K^{\delta}\left(\Omega_{\delta}\right) .
\end{aligned}
$$


By definition, the first-order shape derivative of the potential deformation energy $\mathcal{E}$ at $\Omega_{0}$ in the direction of $V$, if it exists, is given by the limit

$$
\lim _{\delta \rightarrow 0} \frac{\mathcal{E}\left(\Omega_{\delta} ; W^{\delta}, w^{\delta}\right)-\mathcal{E}\left(\Omega_{0} ; W_{0}, w_{0}\right)}{\delta} .
$$

The questions we are now interested in are whether (22) does exist and how this shape derivative can be expressed in an explicit form.

To state our main result, let us fix some notation. For $U, W \in H^{1}\left(\Omega_{0}\right)^{2}$, we define the bilinear form

$A_{p}(V ; U, W)=\operatorname{div}_{x} V \sigma(U): \varepsilon(W)-\sigma(U): E\left(\nabla_{x} V ; W\right)-\sigma(W): E\left(\nabla_{x} V ; U\right)$,

where the second-order tensor $E$ reads as

$$
E(X ; W)=1 / 2\left(\nabla_{x} W X+\left(\nabla_{x} W X\right)^{\mathrm{T}}\right), \quad X \in \mathbb{R}^{2 \times 2} .
$$

Simultaneously, for $u, w \in H^{2}\left(\Omega_{0}\right)$, we put

$$
\begin{gathered}
A_{v}(V ; u, w)=-\operatorname{div}_{x} V m(u): \nabla_{x}^{2} w+m(u):\left(2 \nabla_{x}^{2} w \nabla_{x} V+N \nabla_{x} w\right) \\
+m(w):\left(2 \nabla_{x}^{2} u \nabla_{x} V+N \nabla_{x} u\right),
\end{gathered}
$$

where

$$
\left(N \nabla_{x} w\right)_{i j}=\left(\nabla_{x}^{2} V_{k}\right)_{i j}\left(\nabla_{x} w\right)_{k}
$$

We finally set

$$
\begin{aligned}
& \mathfrak{G}\left(\Omega_{0} ; V\right)=\frac{1}{2} \int_{\Omega \backslash \bar{\omega}_{0}}\left(A_{p}\left(V ; W_{0}, W_{0}\right)+A_{v}\left(V ; w_{0}, w_{0}\right)\right) \mathrm{d} x \\
& \quad-\int_{\Omega \backslash \bar{\omega}_{0}}\left(\operatorname{div}_{x}(F \otimes V) \cdot W_{0}+\operatorname{div}_{x}(f V) w_{0}\right) \mathrm{d} x \\
&-\left\langle\sigma_{\nu_{0}}^{+}\left(W_{0}\right), \nabla_{x} V \llbracket W_{0} \rrbracket \cdot v_{0}\right\rangle_{1 / 2, \gamma_{0}}^{00} \\
&-\left\langle m_{v_{0}}^{+}\left(w_{0}\right), \llbracket \nabla_{x} w_{0} \rrbracket \cdot\left(\nabla_{x} V+\left(\nabla_{x} V\right)^{\mathrm{T}}\right) \nu_{0}\right\rangle_{1 / 2, \gamma_{0}}^{00} \\
&-\left\langle\sigma^{+}\left(W_{0}\right) \nu_{0}, B_{0} V\right\rangle_{1 / 2, \partial \omega_{0}}+\left\langle t_{\nu_{0}}^{+}\left(w_{0}\right), A_{0} \cdot V\right\rangle_{3 / 2, \partial \omega_{0}} \\
&-\left\langle m_{v_{0}}^{+}\left(w_{0}\right), \nabla_{x}\left(A_{0} \cdot V\right) \cdot v_{0}\right\rangle_{1 / 2, \partial \omega_{0}} \\
&-\int_{\omega_{0}}\left(F \cdot B_{0} V+f A_{0} \cdot V\right) \mathrm{d} x .
\end{aligned}
$$


Theorem 2.2 Under the assumptions of Proposition 2.1, the limit in (22) exists and is equal to (25).

The remainder of the paper is devoted to the quite long and technical proof of Theorem 2.2. The key idea behind the proof consists in applying the mapping $T_{\delta}: \Omega_{0} \rightarrow \Omega_{\delta}$ to transform the potential deformation energy $\mathcal{E}\left(\Omega_{\delta} ; W^{\delta}, w^{\delta}\right)$ to the reference domain $\Omega_{0}$ and calculating the desired limit as $\delta \rightarrow 0$ in $\Omega_{0}$. The particular steps are presented below.

We begin with the study of properties of the mapping $T_{\delta}: \Omega_{0} \rightarrow \Omega_{\delta}$. In view of [38, Chapter 2, Lemma 3.4], it generates an isomorphism between the spaces $H_{\partial \Omega}^{1}\left(\Omega_{\delta}\right)^{2} \times$ $H_{\partial \Omega}^{2}\left(\Omega_{\delta}\right)$ and $H_{\partial \Omega}^{1}\left(\Omega_{0}\right)^{2} \times H_{\partial \Omega}^{2}\left(\Omega_{0}\right)$ via

$$
\mathbf{T}_{\delta}: H_{\partial \Omega}^{1}\left(\Omega_{\delta}\right)^{2} \times H_{\partial \Omega}^{2}\left(\Omega_{\delta}\right) \rightarrow H_{\partial \Omega}^{1}\left(\Omega_{0}\right)^{2} \times H_{\partial \Omega}^{2}\left(\Omega_{0}\right):(W, w) \mapsto(W, w) \circ T_{\delta}
$$

For $\left(U^{\delta}, u^{\delta}\right) \in H^{1}\left(\Omega_{\delta}\right)^{2} \times H^{2}\left(\Omega_{\delta}\right)$, we introduce the notation

$$
\left(U_{\delta}, u_{\delta}\right)(x)=\left(U^{\delta}, u^{\delta}\right)\left(T_{\delta}(x)\right), \quad x \in \Omega_{0}
$$

Then the derivatives of first and second orders of such transformed functions can be calculated from the relations

$$
\begin{gathered}
\nabla_{x} U_{\delta}(x)=\nabla_{y} U^{\delta}\left(T_{\delta}(x)\right) \nabla_{x} T_{\delta}(x), \\
\nabla_{x}^{2} u_{\delta}(x)=\left(\nabla_{x} T_{\delta}(x)\right)^{\mathrm{T}} \nabla_{y}^{2} u^{\delta}\left(T_{\delta}(x)\right) \nabla_{x} T_{\delta}(x)+\left(L^{\delta} \nabla_{y} u^{\delta}\right)\left(T_{\delta}(x)\right),
\end{gathered}
$$

where

$$
\left(L^{\delta} \nabla_{y} u^{\delta}\right)_{i j}\left(T_{\delta}(x)\right)=\left(\nabla_{x}^{2} T_{\delta k}(x)\right)_{i j}\left(\nabla_{y} u^{\delta}\right)_{k}\left(T_{\delta}(x)\right)
$$

On the other hand, for $\left(U_{\delta}, u_{\delta}\right) \in H^{1}\left(\Omega_{0}\right)^{2} \times H^{2}\left(\Omega_{0}\right)$, with

$$
\left(U^{\delta}, u^{\delta}\right)(y)=\left(U_{\delta}, u_{\delta}\right)\left(T^{-1}(y)\right), \quad y \in \Omega_{\delta}
$$

it holds

$$
\begin{gathered}
\nabla_{y} U^{\delta}(y)=\nabla_{x} U_{\delta}\left(T_{\delta}^{-1}(y)\right)\left(\nabla_{x} T_{\delta}\right)^{-1}\left(T_{\delta}^{-1}(y)\right), \nabla_{y}^{2} u^{\delta}(y)=\left(\nabla_{x} T_{\delta}\right)^{-\mathrm{T}}\left(T_{\delta}^{-1}(y)\right) \\
\left(\nabla_{x}^{2} u_{\delta}\left(T_{\delta}^{-1}(y)\right)-\left(M_{\delta} \nabla_{x} u_{\delta}\right)\left(T_{\delta}^{-1}(y)\right)\right)\left(\nabla_{x} T_{\delta}\right)^{-1}\left(T_{\delta}^{-1}(y)\right),
\end{gathered}
$$

where

$$
\left(M_{\delta} \nabla_{x} u_{\delta}\right)_{i j}\left(T_{\delta}^{-1}(y)\right)=\left(\nabla_{x}^{2} T_{\delta k}\right)_{i j}\left(T_{\delta}^{-1}(y)\right)\left(\nabla_{x} T_{\delta}\right)_{l k}^{-1}\left(T_{\delta}^{-1}(y)\right)\left(\nabla_{x} u_{\delta}\right)_{l}\left(T^{-1}(y)\right) .
$$

Let $K_{\delta}\left(\Omega_{0}\right)$ be the image of the set of the admissible displacement fields $K^{\delta}\left(\Omega_{\delta}\right)$ under the mapping $\mathbf{T}_{\delta}$. In order to identify $K_{\delta}\left(\Omega_{0}\right)$, we first note that any element 
$(W, w) \in K_{\delta}\left(\Omega_{0}\right)$ satisfies the inequality constraint

$$
\llbracket W \rrbracket \cdot v_{\delta} \geq h\left|\left(\nabla_{x} T_{\delta}\right)^{-\mathrm{T}} \llbracket \nabla_{x} w \rrbracket \cdot v_{\delta}\right| \quad \text { on } \quad \gamma_{0} .
$$

The transformed outward pointing unit normal $v_{\delta}=v^{\delta} \circ T_{\delta}$ is related to $v_{0}$ through the equation [6, Section 1.7]

$$
v_{\delta}=\frac{\left(\nabla_{x} T_{\delta}\right)^{-\mathrm{T}} v_{0}}{\left\|\left(\nabla_{x} T_{\delta}\right)^{-\mathrm{T}} v_{0}\right\|_{2}},
$$

where $\|\cdot\|_{2}$ stands for the Euclidean norm in $\mathbb{R}^{2}$. Inequality (26) then reads

$$
\llbracket W \rrbracket \cdot\left(\nabla_{x} T_{\delta}\right)^{-\mathrm{T}} v_{0} \geq h\left|\llbracket \nabla_{x} w \rrbracket \cdot\left(\nabla_{x} T_{\delta}\right)^{-1}\left(\nabla_{x} T_{\delta}\right)^{-\mathrm{T}} v_{0}\right| \quad \text { on } \quad \gamma_{0} .
$$

Additionally, the restriction $(W, w)$ on the domain $\omega_{0}$ belongs to the Cartesian product $R_{p}^{\delta}\left(\omega_{0}\right) \times R_{v}^{\delta}\left(\omega_{0}\right)$ of the sets

$$
\begin{aligned}
& R_{p}^{\delta}\left(\omega_{0}\right)=\left\{W(x)=B T_{\delta}(x)+C: B \in \mathbb{R}^{2 \times 2}, B=-B^{\mathrm{T}}, C \in \mathbb{R}^{2}, x \in \omega_{0}\right\} \\
& R_{v}^{\delta}\left(\omega_{0}\right)=\left\{w(x)=A \cdot T_{\delta}(x)+a: A \in \mathbb{R}^{2}, a \in \mathbb{R}, x \in \omega_{0}\right\} .
\end{aligned}
$$

Thus, $K_{\delta}\left(\Omega_{0}\right)$ may be defined as follows

$$
\begin{aligned}
K_{\delta}\left(\Omega_{0}\right)=\{ & (W, w) \in H_{\partial \Omega}^{1}\left(\Omega_{0}\right)^{2} \times H_{\partial \Omega}^{2}\left(\Omega_{0}\right):\left.(W, w)\right|_{\omega_{0}} \in R_{p}^{\delta}\left(\omega_{0}\right) \times R_{v}^{\delta}\left(\omega_{0}\right) ; \\
& \left.\llbracket W \rrbracket \cdot\left(\nabla_{x} T_{\delta}\right)^{-\mathrm{T}} v_{0} \geq h\left|\llbracket \nabla_{x} w \rrbracket \cdot\left(\nabla_{x} T_{\delta}\right)^{-1}\left(\nabla_{x} T_{\delta}\right)^{-\mathrm{T}} v_{0}\right| \text { on } \gamma_{0}\right\}
\end{aligned}
$$

and so $\mathbf{T}_{\delta}$ is a bijection between the sets $K^{\delta}\left(\Omega_{\delta}\right)$ and $K_{\delta}\left(\Omega_{0}\right)$.

The change of variables $y=T_{\delta}(x)$ in the variational inequality (7) implies that $\left(W_{\delta}, w_{\delta}\right) \in K_{\delta}\left(\Omega_{0}\right)$ is a unique solution of the variational inequality

$$
\begin{aligned}
& 2 h \int_{\Omega \backslash \bar{\omega}_{0}} \operatorname{det}\left(\nabla_{x} T_{\delta}\right) \mathbb{C} E\left(\left(\nabla_{x} T_{\delta}\right)^{-1} ; W_{\delta}\right): E\left(\left(\nabla_{x} T_{\delta}\right)^{-1} ; \bar{W}-W_{\delta}\right) \mathrm{d} x \\
& \quad-\frac{2 h^{3}}{3} \int_{\Omega \backslash \bar{\omega}_{0}} \operatorname{det}\left(\nabla_{x} T_{\delta}\right) \mathbb{C}\left\{\left(\nabla_{x} T_{\delta}\right)^{-\mathrm{T}}\left(\nabla_{x}^{2} w_{\delta}-M_{\delta} \nabla_{x} w_{\delta}\right)\left(\nabla_{x} T_{\delta}\right)^{-1}\right\} \\
& \quad:\left\{\left(\nabla_{x} T_{\delta}\right)^{-\mathrm{T}}\left(\nabla_{x}^{2}\left(\bar{w}-w_{\delta}\right)-M_{\delta} \nabla_{x}\left(\bar{w}-w_{\delta}\right)\right)\left(\nabla_{x} T_{\delta}\right)^{-1}\right\} \mathrm{d} x \\
& \geq \int_{\Omega_{0}} \operatorname{det}\left(\nabla_{x} T_{\delta}\right) F_{\delta} \cdot\left(\bar{W}-W_{\delta}\right) \mathrm{d} x+\int_{\Omega_{0}} \operatorname{det}\left(\nabla_{x} T_{\delta}\right) f_{\delta}\left(\bar{w}-w_{\delta}\right) \mathrm{d} x \\
& \forall(\bar{W}, \bar{w}) \in K_{\delta}\left(\Omega_{0}\right) .
\end{aligned}
$$

Here, $\left(F_{\delta}, f_{\delta}\right)(x)=(F, f)\left(T_{\delta}(x)\right)$. 
As a preliminary step towards the verification of the uniform boundedness of $\left(W_{\delta}, w_{\delta}\right)$ in $H_{\partial \Omega}^{1}\left(\Omega_{0}\right)^{2} \times H_{\partial \Omega}^{2}\left(\Omega_{0}\right)$, we establish the next proposition.

Proposition 2.3 The following Taylor formulae as $\delta \rightarrow 0$ are valid:

$$
\begin{array}{r}
T_{\delta}=\operatorname{Id}_{x}+\delta V+r_{\delta}^{1}, \quad\left\|r_{\delta}^{1}\right\|_{W^{2, \infty}(\Omega)^{2}}=o(\delta), \\
\nabla_{x} T_{\delta}=I+\delta \nabla_{x} V+r_{\delta}^{2}, \quad\left\|r_{\delta}^{2}\right\|_{W^{1, \infty}(\Omega)^{2 \times 2}}=o(\delta), \\
\operatorname{det}\left(\nabla_{x} T_{\delta}\right)=1+\delta \operatorname{div}_{x} V+r_{\delta}^{3}, \quad\left\|r_{\delta}^{3}\right\|_{W^{1, \infty}(\Omega)}=o(\delta), \\
\left(\nabla_{x} T_{\delta}\right)^{-1}=I-\delta \nabla_{x} V+r_{\delta}^{4}, \quad\left\|r_{\delta}^{4}\right\|_{W^{1, \infty}(\Omega)^{2 \times 2}}=o(\delta),
\end{array}
$$

Proof This is an immediate consequence of Proposition 2.1.

Lemma 2.4 There exists $\delta_{1} \in\left(0, \delta_{0}\right)$ such that the solution $\left(W_{\delta}, w_{\delta}\right)$ of the transformed variational inequality (27) is uniformly bounded with respect to $\delta \in\left[-\delta_{1}, \delta_{1}\right]$ in $H_{\partial \Omega}^{1}\left(\Omega_{0}\right)^{2} \times H_{\partial \Omega}^{2}\left(\Omega_{0}\right)$ by a constant $c>0$ :

$$
\left\|\left(W_{\delta}, w_{\delta}\right)\right\|_{H^{1}\left(\Omega_{0}\right)^{2} \times H^{2}\left(\Omega_{0}\right)} \leq c .
$$

Proof Since $\left.\left(W^{\delta}, w^{\delta}\right)\right|_{\omega_{\delta}} \in R_{p}\left(\omega_{\delta}\right) \times R_{v}\left(\omega_{\delta}\right)$, we can replace the integration domain $\Omega \backslash \bar{\omega}_{\delta}$ by $\Omega_{\delta}$ in (21) and the integration domain $\Omega \backslash \bar{\omega}_{0}$ by $\Omega_{0}$ in (27). The modified variational inequality (27) is referred to as $(27)_{\Omega_{0}}$. Let $(W, w),(U, u) \in H^{1}\left(\Omega_{0}\right)^{2} \times$ $H^{2}\left(\Omega_{0}\right)$. Invoking Proposition 2.3, we deduce that the integrals on the left-hand side of $(27)_{\Omega_{0}}$ admit the following asymptotic expansions as $\delta \rightarrow 0$ :

$$
\begin{aligned}
& 2 h \int_{\Omega_{0}} \operatorname{det}\left(\nabla_{x} T_{\delta}\right) \mathbb{C} E\left(\left(\nabla_{x} T_{\delta}\right)^{-1} ; U\right): E\left(\left(\nabla_{x} T_{\delta}\right)^{-1} ; W\right) \mathrm{d} x \\
& =\int_{\Omega_{0}}\left(\sigma(U): \varepsilon(W)+\delta A_{p}(V ; U, W)+R_{1}(\delta, U, W)\right) \mathrm{d} x, \\
& \frac{2 h^{3}}{3} \int_{\Omega_{0}} \operatorname{det}\left(\nabla_{x} T_{\delta}\right) \mathbb{C}\left\{\left(\nabla_{x} T_{\delta}\right)^{-\mathrm{T}}\left(\nabla_{x}^{2} u-M_{\delta} \nabla_{x} u\right)\left(\nabla_{x} T_{\delta}\right)^{-1}\right\} \\
& :\left\{\left(\nabla_{x} T_{\delta}\right)^{-\mathrm{T}}\left(\nabla_{x}^{2} w-M_{\delta} \nabla_{x} w\right)\left(\nabla_{x} T_{\delta}\right)^{-1}\right\} \mathrm{d} x \\
& =\int_{\Omega_{0}}\left(-m(u): \nabla_{x}^{2} w+\delta A_{v}(V ; u, w)+R_{2}(\delta, u, w)\right) \mathrm{d} x,
\end{aligned}
$$

where

$$
\begin{aligned}
& \left\|R_{1}(\delta, U, W)\right\|_{L^{1}\left(\Omega_{0}\right)} \leq c_{1}(|\delta|)\|U\|_{H^{1}\left(\Omega_{0}\right)^{2}}\|W\|_{H^{1}\left(\Omega_{0}\right)^{2}}, \quad 0 \leq c_{1}(|\delta|)=o(\delta) \\
& \left\|R_{2}(\delta, u, w)\right\|_{L^{1}\left(\Omega_{0}\right)} \leq c_{2}(|\delta|)\|u\|_{H^{2}\left(\Omega_{0}\right)}\|w\|_{H^{2}\left(\Omega_{0}\right)}, \quad 0 \leq c_{2}(|\delta|)=o(\delta)
\end{aligned}
$$


The terms in the right-hand side of $(27)_{\Omega_{0}}$ can be rewritten in like manner:

$$
\begin{aligned}
& \int_{\Omega_{0}} \operatorname{det}\left(\nabla_{x} T_{\delta}\right) F_{\delta} \cdot W \mathrm{~d} x=\int_{\Omega_{0}}\left(F \cdot W+\delta \operatorname{div}_{x}(F \otimes V) \cdot W+R_{3}(\delta, W)\right) \mathrm{d} x,(37) \\
& \int_{\Omega_{0}} \operatorname{det}\left(\nabla_{x} T_{\delta}\right) f_{\delta} w \mathrm{~d} x=\int_{\Omega_{0}}\left(f w+\delta \operatorname{div}_{x}(f V) w+R_{4}(\delta, w)\right) \mathrm{d} x
\end{aligned}
$$

where

$$
\begin{gathered}
\left\|R_{3}(\delta, W)\right\|_{L^{1}\left(\Omega_{0}\right)} \leq c_{3}(|\delta|)\|W\|_{L^{2}\left(\Omega_{0}\right)^{2}}, \quad 0 \leq c_{3}(|\delta|)=o(\delta) \\
\left\|R_{4}(\delta, w)\right\|_{L^{1}\left(\Omega_{0}\right)} \leq c_{4}(|\delta|)\|w\|_{L^{2}\left(\Omega_{0}\right)}, \quad 0 \leq c_{4}(|\delta|)=o(\delta) .
\end{gathered}
$$

Moreover, since $\nabla_{x} V \in C^{0,1}(\bar{\Omega})^{2 \times 2}$ and $(F, f) \in C^{1}(\bar{\Omega})^{2} \times C^{1}(\bar{\Omega})$, we arrive at the estimates

$$
\begin{gathered}
\left\|A_{p}(V ; U, W)\right\|_{L^{1}\left(\Omega_{0}\right)} \leq c\|U\|_{H^{1}\left(\Omega_{0}\right)^{2}}\|W\|_{H^{1}\left(\Omega_{0}\right)^{2}}, \\
\left\|A_{v}(V ; u, w)\right\|_{L^{1}\left(\Omega_{0}\right)} \leq c\|u\|_{H^{2}\left(\Omega_{0}\right)}\|w\|_{H^{2}\left(\Omega_{0}\right)}, \\
\left\|\operatorname{div}_{x}(F \otimes V) \cdot W\right\|_{L^{1}\left(\Omega_{0}\right)} \leq c\|W\|_{L^{2}\left(\Omega_{0}\right)^{2}} \\
\left\|\operatorname{div}_{x}(f V) w\right\|_{L^{1}\left(\Omega_{0}\right)} \leq c\|w\|_{L^{2}\left(\Omega_{0}\right)} .
\end{gathered}
$$

We next take $\bar{W}=(0,0)^{\mathrm{T}}$ and $\bar{w}=0$ into the variational inequality $(27)_{\Omega_{0}}$ and use the asymptotic expansions (33)-(34) and (37)-(38) to discover

$$
\begin{aligned}
\int_{\Omega_{0}} \sigma\left(W_{\delta}\right): \varepsilon\left(W_{\delta}\right) \mathrm{d} x-\int_{\Omega_{0}} m\left(w_{\delta}\right): \nabla_{x}^{2} w_{\delta} \mathrm{d} x \\
\leq-\delta \int_{\Omega_{0}}\left(A_{p}\left(V ; W_{\delta}, W_{\delta}\right)+A_{v}\left(V ; w_{\delta}, w_{\delta}\right)\right) \mathrm{d} x \\
\quad+\int_{\Omega_{0}}\left(F \cdot W_{\delta}+f w_{\delta}+\delta\left(\operatorname{div}_{x}(F \otimes V) \cdot W_{\delta}+\operatorname{div}_{x}(f V) w_{\delta}\right)-R_{1}\left(\delta, W_{\delta}, W_{\delta}\right)\right. \\
\left.\quad-R_{2}\left(\delta, w_{\delta}, w_{\delta}\right)+R_{3}\left(\delta, W_{\delta}\right)+R_{4}\left(\delta, w_{\delta}\right)\right) \mathrm{d} x .
\end{aligned}
$$

Applying the Korn inequality and the Friedrichs inequality on the left-hand side of (43), the Cauchy inequality and estimates (35)-(36), (39)-(40), and (41)-(42) on the right-hand side of (43) yields that there exists $\delta_{1} \in\left(0, \delta_{0}\right)$ such that for every $\delta \in\left[-\delta_{1}, \delta_{1}\right]$ the uniform estimate (32) takes place. This proofs the lemma.

For convenience purposes, we collect here auxiliary statements that will be employed repeatedly in the sequel. 
Proposition 2.5 [19, Lemma 1.14] Let $u \in H^{1 / 2}\left(\partial \omega_{0}\right)$ and $v \in C^{0,1}\left(\partial \omega_{0}\right)$. Then $u v \in H^{1 / 2}\left(\partial \omega_{0}\right)$ and

$$
\|u v\|_{H^{1 / 2}\left(\partial \omega_{0}\right)} \leq c\|u\|_{H^{1 / 2}\left(\partial \omega_{0}\right)}\|v\|_{C^{0,1}\left(\partial \omega_{0}\right)}
$$

with $c>0$ independent of $u$ and $v$.

We label the part $\partial \Omega \cup \partial \omega_{0} \backslash \gamma_{0}$ of the outer boundary of the domain $\Omega_{0} \backslash \bar{\omega}_{0}$ as $\Sigma$. For each $k=1,2$, we introduce the extension operator $\operatorname{Ext}_{k}: H_{\Sigma}^{k}\left(\Omega \backslash \bar{\omega}_{0}\right)^{k_{*}} \rightarrow$ $H_{\partial \Omega}^{k}\left(\Omega_{0}\right)^{k_{*}}$ given by

$$
v \mapsto \begin{cases}(\underbrace{0, \ldots, 0}_{k_{*}})^{\mathrm{T}} & \text { in } \omega_{0}, \\ v & \text { in } \Omega \backslash \bar{\omega}_{0} .\end{cases}
$$

It is clearly that $\mathrm{Ext}_{k}$ are well defined, linear, and continuous operators.

With these preliminaries behind us, we are able to state the key assertion of our analysis that guarantees the existence of certain test elements.

Theorem 2.6 Let $\left(W_{0}, w_{0}\right) \in K_{0}\left(\Omega_{0}\right)$ be the solution of the variational inequality (7), and let $\left(W_{\delta}, w_{\delta}\right) \in K_{\delta}\left(\Omega_{0}\right)$ be the solution of the transformed variational inequality (27). Then there exists $\delta_{2} \in\left(0, \delta_{1}\right]$ and tuples $\left(W_{\delta}^{i}, w_{\delta}^{i}\right)$ such that for every $\delta \in$ $\left[-\delta_{2}, \delta_{2}\right]$ the inclusions

$$
\begin{aligned}
& \left(\widehat{W}_{\delta}^{1}, \widehat{w}_{\delta}^{1}\right)=\left(W_{0}, w_{0}\right)+\delta\left(W_{\delta}^{1}, w_{\delta}^{1}\right) \in K_{\delta}\left(\Omega_{0}\right), \\
& \left(\widehat{W}_{\delta}^{2}, \widehat{w}_{\delta}^{2}\right)=\left(W_{\delta}, w_{\delta}\right)-\delta\left(W_{\delta}^{2}, w_{\delta}^{2}\right) \in K_{0}\left(\Omega_{0}\right),
\end{aligned}
$$

and uniform in $\delta$ bounds

$$
\left\|\left(W_{\delta}^{i}, w_{\delta}^{i}\right)\right\|_{H^{1}\left(\Omega_{0}\right)^{2} \times H^{2}\left(\Omega_{0}\right)} \leq c, \quad i=1,2,
$$

are valid.

Proof For each $i=1,2$, we construct the required tuple $\left(W_{\delta}^{i}, w_{\delta}^{i}\right)$ as sums $W_{\delta}^{i}=$ $P_{\delta}^{i}+Q_{\delta}^{i}$ and $w_{\delta}^{i}=p_{\delta}^{i}+q_{\delta}^{i}$, where the set $\left\{P_{\delta}^{i}, p_{\delta}^{i}\right\}$ provides the appropriate inequality constraint for $\left(\widehat{W}_{\delta}^{i}, \widehat{w}_{\delta}^{i}\right)$ on $\gamma_{0}$, and the set $\left\{Q_{\delta}^{i}, q_{\delta}^{i}\right\}$ is responsible for the correct structure of $\left(\widehat{W}_{\delta}^{i}, \widehat{w}_{\delta}^{i}\right)$ in $\omega_{0}$, which is specified by the equality constraint. The case $\delta=0$ is trivial, so we assume that $\delta \neq 0$.

We start with the construction of $\left(W_{\delta}^{1}, w_{\delta}^{1}\right)$. Since $\llbracket W_{0} \rrbracket \in H^{1 / 2}\left(\partial \omega_{0}\right)^{2}$ and by Proposition 2.5, the function

$$
P_{\delta}^{1}=\operatorname{Ext}_{1} \circ \operatorname{Tr}_{1, \Omega \backslash \bar{\omega}_{0}}^{-1}\left(\frac{\nabla_{x} T_{\delta}-I}{\delta} \llbracket W_{0} \rrbracket ;(0,0)^{\mathrm{T}}\right)
$$

belongs to $H_{\partial \Omega}^{1}\left(\Omega_{0}\right)^{2}$. We define $w_{\delta}^{1}$ in the following way. Observe first that $Z_{\delta}=$ $\left(\nabla_{x} T_{\delta}\right)^{-1}\left(\nabla_{x} T_{\delta}\right)^{-\mathrm{T}} \in C^{0,1}(\bar{\Omega})^{2 \times 2}$. For any function $v \in H^{2}\left(\Omega \backslash \bar{\omega}_{0}\right)$, we write

$\left(\left(\nabla_{x} v\right)^{+} \cdot v_{0}\right) v_{0} \cdot Z_{\delta} v_{0}=\left(\nabla_{x} v\right)^{+} \cdot Z_{\delta} v_{0}+\left(\nabla_{x} v\right)^{+} \cdot\left(v_{0} \otimes v_{0} Z_{\delta}-Z_{\delta}\right) v_{0} \quad$ on $\partial \omega_{0}$. 
There exists $\delta_{2} \in\left(0, \delta_{1}\right]$ and $\alpha>0$ such that $\alpha_{\delta}(s)=\left(v_{0} \cdot Z_{\delta} \nu_{0}\right)(s) \geq \alpha$ for every $(\delta, s) \in\left[-\delta_{2}, \delta_{2}\right] \times \partial \omega_{0}$. For $Z_{\delta \tau_{0}}=\left(\nu_{0} \otimes v_{0} Z_{\delta}-Z_{\delta}\right) \nu_{0}$, we compute $v_{0} \cdot Z_{\delta \tau_{0}}=0$ on $\partial \omega_{0}$, which implies $Z_{\delta \tau_{0}}=\beta_{\delta} \tau_{0}$ with $\beta_{\delta}=\tau_{0} \cdot Z_{\delta \tau_{0}}$. By construction, $\beta_{\delta} \in C^{0,1}\left(\partial \omega_{0}\right)$. With the help of standard arguments in local coordinates (see also [11, Proposition 3.1]), we infer

$$
\left(\nabla_{x} v\right)^{+}=\left(\partial_{\tau_{0}} v^{+}\right) \tau_{0}+\left(\left(\nabla_{x} v\right)^{+} \cdot v_{0}\right) v_{0} \quad \text { on } \quad \partial \omega_{0}
$$

and so

$$
\alpha_{\delta}\left(\nabla_{x} v\right)^{+} \cdot v_{0}=\left(\nabla_{x} v\right)^{+} \cdot Z_{\delta} v_{0}+\beta_{\delta} \partial_{\tau_{0}} v^{+} \text {on } \partial \omega_{0}
$$

Exploiting the inclusions $\llbracket w_{0} \rrbracket \in H^{3 / 2}\left(\partial \omega_{0}\right)$ and $\llbracket \nabla_{x} w_{0} \rrbracket \in H^{1 / 2}\left(\partial \omega_{0}\right)^{2}$ and once more Proposition 2.5, we conclude that the function

$$
p_{\delta}^{1}=\operatorname{Ext}_{2} \circ \operatorname{Tr}_{2, \Omega \backslash \bar{\omega}_{0}}^{-1}\left(\llbracket w_{0} \rrbracket ; 0 ; \frac{1}{\alpha_{\delta}}\left(\llbracket \nabla_{x} w_{0} \rrbracket \cdot \frac{I-Z_{\delta}}{\delta} v_{0}+\beta_{\delta} \partial_{\tau_{0}} \llbracket w_{0} \rrbracket\right) ; 0\right)
$$

is from the space $H_{\partial \Omega}^{2}\left(\Omega_{0}\right)$. Under the circumstances, a straightforward calculation reveals

$$
\begin{aligned}
\llbracket \nabla_{x} p_{\delta}^{1} \rrbracket \cdot Z_{\delta} v_{0} & =\left(\nabla_{x} p_{\delta}^{1}\right)^{+} \cdot Z_{\delta} v_{0}=\alpha_{\delta}\left(\nabla_{x} p_{\delta}^{1}\right)^{+} \cdot v_{0}-\beta_{\delta} \partial_{\tau_{0}} p_{\delta}^{1+} \\
& =\llbracket \nabla_{x} w_{0} \rrbracket \cdot \frac{I-Z_{\delta}}{\delta} v_{0} \quad \text { on } \partial \omega_{0} .
\end{aligned}
$$

Let us set

$$
Q_{\delta}^{1}(x)=B_{0} \frac{T_{\delta}-\operatorname{Id}_{x}}{\delta}(x), \quad q_{\delta}^{1}(x)=A_{0} \cdot \frac{T_{\delta}-\operatorname{Id}_{x}}{\delta}(x), \quad x \in \Omega .
$$

The functions $Q_{\delta}^{1}$ and $q_{\delta}^{1}$ vanish in a neighborhood of $\partial \Omega$. We now check that $\left(\widehat{W}_{\delta}^{1}, \widehat{w}_{\delta}^{1}\right) \in K_{\delta}\left(\Omega_{0}\right)$. In fact, the inequality constraint follows from the following chain of equalities and inequalities

$$
\begin{aligned}
\llbracket \widehat{W}_{\delta}^{1} \rrbracket \cdot\left(\nabla_{x} T_{\delta}\right)^{-\mathrm{T}} v_{0} & =\llbracket W_{0}+\delta P_{\delta}^{1} \rrbracket \cdot\left(\nabla_{x} T_{\delta}\right)^{-\mathrm{T}} v_{0} \\
& \stackrel{(45)}{=}\left(\llbracket W_{0} \rrbracket+\left(\nabla_{x} T_{\delta}-I\right) \llbracket W_{0} \rrbracket\right) \cdot\left(\nabla_{x} T_{\delta}\right)^{-\mathrm{T}} v_{0}=\llbracket W_{0} \rrbracket \cdot v_{0} \\
& \geq h\left|\llbracket \nabla_{x} w_{0} \rrbracket \cdot v_{0}\right|=h\left|\llbracket \nabla_{x} w_{0} \rrbracket \cdot\left(I-Z_{\delta}+Z_{\delta}\right) v_{0}\right| \\
& \stackrel{(46)}{=} h|\llbracket| \nabla_{x}\left(w_{0}+\delta p_{\delta}^{1}\right) \rrbracket \cdot Z_{\delta} v_{0}|=h| \llbracket \nabla_{x} \widehat{w}_{\delta}^{1} \rrbracket \cdot Z_{\delta} v_{0} \mid \text { on } \gamma_{0} .
\end{aligned}
$$

Moreover, we have no difficulty in verifying the equality constraint,

$$
\begin{aligned}
\widehat{W}_{\delta}^{1}(x) & =W_{0}(x)+\delta Q_{\delta}^{1}(x)=B_{0} \operatorname{Id}_{x}(x)+C_{0}+B_{0}\left(T_{\delta}(x)-\operatorname{Id}_{x}(x)\right) \\
& =B_{0} T_{\delta}(x)+C_{0} \text { in } \omega_{0},
\end{aligned}
$$




$$
\begin{aligned}
\widehat{w}_{\delta}^{1}(x) & =w_{0}(x)+\delta q_{\delta}^{1}(x)=A_{0} \cdot \operatorname{Id}_{x}(x)+a_{0}+A_{0} \cdot\left(T_{\delta}(x)-\operatorname{Id}_{x}(x)\right) \\
& =A_{0} \cdot T_{\delta}(x)+a_{0} \text { in } \omega_{0} .
\end{aligned}
$$

We next turn our attention to $\left(W_{\delta}^{2}, w_{\delta}^{2}\right)$. The construction of $W_{\delta}^{2}$ is not much harder than that for $W_{\delta}^{1}$. Indeed, with $\llbracket W_{\delta} \rrbracket \in H^{1 / 2}\left(\partial \omega_{0}\right)$ and Proposition 2.5 in mind, the function

$$
P_{\delta}^{2}=\operatorname{Ext}_{1} \circ \operatorname{Tr}_{1, \Omega \backslash \bar{\omega}_{0}}^{-1}\left(\frac{I-\left(\nabla_{x} T_{\delta}\right)^{-1}}{\delta} \llbracket W_{\delta} \rrbracket ;(0,0)^{\mathrm{T}}\right)
$$

is an element of the space $H_{\partial \Omega}^{1}\left(\Omega_{0}\right)^{2}$. To define $w_{\delta}^{2}$, we appeal to slightly simpler arguments than for $w_{\delta}^{1}$. Taking into account the inclusions $\llbracket w_{\delta} \rrbracket \in H^{3 / 2}\left(\partial \omega_{0}\right)$ and $\llbracket \nabla_{x} w_{\delta} \rrbracket \in H^{1 / 2}\left(\partial \omega_{0}\right)^{2}$ and applying Proposition 2.5 again, we derive that the function

$$
p_{\delta}^{2}=\operatorname{Ext}_{2} \circ \operatorname{Tr}_{2, \Omega \backslash \bar{\omega}_{0}}^{-1}\left(\llbracket w_{\delta} \rrbracket ; 0 ; \llbracket \nabla_{x} w_{\delta} \rrbracket \cdot \frac{I-Z_{\delta}}{\delta} v_{0} ; 0\right)
$$

belongs to $H_{\partial \Omega}^{2}\left(\Omega_{0}\right)$. We finally put

$$
Q_{\delta}^{2}(x)=B_{\delta} \frac{T_{\delta}-\mathrm{Id}_{x}}{\delta}(x), \quad q_{\delta}^{2}=A_{\delta} \cdot \frac{T_{\delta}-\mathrm{Id}_{x}}{\delta}(x), \quad x \in \Omega
$$

The functions $Q_{\delta}^{2}$ and $q_{\delta}^{2}$ vanish in a neighborhood of $\partial \Omega$. In view of the above discussion, we obtain

$$
\begin{aligned}
\llbracket \widehat{W}_{\delta}^{2} \rrbracket \cdot v_{0} & =\llbracket W_{\delta}-\delta P_{\delta}^{2} \rrbracket \cdot v_{0} \stackrel{(47)}{=}\left(\llbracket W_{\delta} \rrbracket-\left(I-\left(\nabla_{x} T_{\delta}\right)^{-1}\right) \llbracket W_{\delta} \rrbracket\right) \cdot v_{0} \\
& =\llbracket W_{\delta} \rrbracket \cdot\left(\nabla_{x} T_{\delta}\right)^{-\mathrm{T}} v_{0} \geq h\left|\llbracket \nabla_{x} w_{\delta} \rrbracket \cdot Z_{\delta} v_{0}\right| \stackrel{(48)}{=} h\left|\llbracket \nabla_{x}\left(w_{\delta}-\delta p_{\delta}^{2}\right) \rrbracket \cdot v_{0}\right| \\
& =h\left|\llbracket \nabla_{x} \widehat{w}_{\delta}^{2} \rrbracket \cdot v_{0}\right| \quad \text { on } \quad \gamma_{0} .
\end{aligned}
$$

Furthermore,

$$
\begin{aligned}
\widehat{W}_{\delta}^{2}(x) & =W_{\delta}(x)-\delta Q_{\delta}^{2}(x)=B_{\delta} T_{\delta}(x)+C_{\delta}-B_{\delta}\left(T_{\delta}(x)-\operatorname{Id}_{x}(x)\right) \\
& =B_{\delta} \operatorname{Id}_{x}(x)+C_{\delta} \text { in } \omega_{0}, \\
\widehat{w}_{\delta}^{2}(x) & =w_{\delta}(x)-\delta q_{\delta}^{2}(x)=A_{\delta} \cdot T_{\delta}(x)+a_{\delta}-A_{\delta} \cdot\left(T_{\delta}(x)-\operatorname{Id}_{x}(x)\right) \\
& =A_{\delta} \cdot \operatorname{Id}_{x}(x)+a_{\delta} \text { in } \omega_{0} .
\end{aligned}
$$

We thus can conclude that $\left(\widehat{W}_{\delta}^{2}, \widehat{w}_{\delta}^{2}\right) \in K_{0}\left(\Omega_{0}\right)$, as asserted.

In order to complete the proof, we observe that the uniform estimates (44) is an immediate consequence of (32) and continuity of the lifting and extension operators.

These considerations allow us to establish convergence properties of the transformed solutions $\left(W_{\delta}, w_{\delta}\right)$ as $\delta \rightarrow 0$. 
Lemma 2.7 There exists a constant $c>0$ that is independent of $\delta$ such that it holds for every $\delta \in\left[-\delta_{2}, \delta_{2}\right]$ :

$$
\left\|\left(W_{\delta}, w_{\delta}\right)-\left(W_{0}, w_{0}\right)\right\|_{H^{1}\left(\Omega_{0}\right)^{2} \times H^{2}\left(\Omega_{0}\right)} \leq c \sqrt{|\delta|} .
$$

Proof As in the proof of Lemma 2.4, we change the integration domain $\Omega \backslash \bar{\omega}_{0}$ by $\Omega_{0}$ in the variational inequality (7). The resulting variational inequality is referred to as $(7)_{\Omega_{0}}$. Inserting $\left(\widehat{W}_{\delta}^{1}, \widehat{w}_{\delta}^{1}\right)$ and $\left(\widehat{W}_{\delta}^{2}, \widehat{w}_{\delta}^{2}\right)$ as test functions for $(27)_{\Omega_{0}}$ and $(7)_{\Omega_{0}}$, respectively, and adding both variational inequalities, we invoke the asymptotic expansions (33)(34) and (37)-(38) to obtain

$$
\begin{aligned}
\int_{\Omega_{0}} \sigma & \left(W_{\delta}-W_{0}\right): \varepsilon\left(W_{\delta}-W_{0}\right) \mathrm{d} x-\int_{\Omega_{0}} m\left(w_{\delta}-w_{0}\right): \nabla_{x}^{2}\left(w_{\delta}-w_{0}\right) \mathrm{d} x \\
\leq & \delta \int_{\Omega_{0}}\left(A_{p}\left(V ; W_{\delta}, W_{0}+\delta W_{\delta}^{1}-W_{\delta}\right)+A_{v}\left(V ; w_{\delta}, w_{0}+\delta w_{\delta}^{1}-w_{\delta}\right)\right) \mathrm{d} x \\
& +\delta \int_{\Omega_{0}}\left(F \cdot\left(W_{\delta}^{2}-W_{\delta}^{1}\right)+f\left(w_{\delta}^{2}-w_{\delta}^{1}\right)-\operatorname{div}_{x}(F \otimes V) \cdot\left(W_{0}+\delta W_{\delta}^{1}-W_{\delta}\right)\right. \\
& \left.-\operatorname{div}_{x}(f V)\left(w_{0}+\delta w_{\delta}^{1}-w_{\delta}\right)\right) \mathrm{d} x \\
& +\delta \int_{\Omega_{0}}\left(\sigma\left(W_{\delta}\right): \varepsilon\left(W_{\delta}^{1}\right)-m\left(w_{\delta}\right): \nabla_{x}^{2} w_{\delta}^{1}\right) \mathrm{d} x \\
& -\delta \int_{\Omega_{0}}\left(\sigma\left(W_{0}\right): \varepsilon\left(W_{\delta}^{2}\right)-m\left(w_{0}\right): \nabla_{x}^{2} w_{\delta}^{2}\right) \mathrm{d} x \\
& +\int_{\Omega_{0}}\left(R_{1}\left(\delta, W_{\delta}, W_{0}+\delta W_{\delta}^{1}-W_{\delta}\right)+R_{2}\left(\delta, w_{\delta}, w_{0}+\delta w_{\delta}^{1}-w_{\delta}\right)\right) \mathrm{d} x \\
& +\int_{\Omega_{0}}\left(R_{3}\left(\delta, W_{0}+\delta W_{\delta}^{1}-W_{\delta}\right)+R_{4}\left(\delta, w_{\delta}, w_{0}+\delta w_{\delta}^{1}-w_{\delta}\right)\right) \mathrm{d} x .
\end{aligned}
$$

By virtue of estimates (32),(35)-(36),(39)-(42), and (44), it holds for every $\delta \in$ $\left[-\delta_{2}, \delta_{2}\right]$ :

$$
\int_{\Omega_{0}} \sigma\left(W_{\delta}-W_{0}\right): \varepsilon\left(W_{\delta}-W_{0}\right) \mathrm{d} x-\int_{\Omega_{0}} m\left(w_{\delta}-w_{0}\right): \nabla_{x}^{2}\left(w_{\delta}-w_{0}\right) \mathrm{d} x \leq c|\delta| .
$$

We finally apply the Korn inequality and the Friedrichs inequality to complete the proof.

Corollary 2.8 The sequence $\left(B_{\delta}, C_{\delta}, A_{\delta}, a_{\delta}\right)$ converges to $\left(B_{0}, C_{0}, A_{0}, a_{0}\right)$ in $\mathbb{R}^{2 \times 2} \times$ $\mathbb{R}^{2} \times \mathbb{R}^{2} \times \mathbb{R}$ as $\delta \rightarrow 0$. 
Proof By Lemma 2.7, it follows that $\left(W_{\delta}, w_{\delta}\right) \rightarrow\left(W_{0}, w_{0}\right)$ strongly in $H^{1}\left(\omega_{0}\right)^{2} \times$ $H^{2}\left(\omega_{0}\right)$, in other words,

$$
\begin{aligned}
& B_{\delta} \operatorname{Id}_{x}+C_{\delta}+\delta B_{\delta}\left(V+\frac{r_{\delta}^{1}}{\delta}\right) \rightarrow B_{0} \operatorname{Id}_{x}+C_{0} \text { strongly in } H^{1}\left(\omega_{0}\right)^{2}, \\
& A_{\delta} \cdot \mathrm{Id}_{x}+a_{\delta}+\delta A_{\delta} \cdot\left(V+\frac{r_{\delta}^{1}}{\delta}\right) \rightarrow A_{0} \cdot \mathrm{Id}_{x}+a_{0} \text { strongly in } H^{2}\left(\omega_{0}\right) .
\end{aligned}
$$

Estimate (32) forces that the sequences $B_{\delta}$ and $A_{\delta}$ are uniformly bounded with respect to small enough $\delta$. Thus we can rewrite (49)-(50) as

$$
\begin{aligned}
& B_{\delta} \operatorname{Id}_{x}+C_{\delta} \rightarrow B_{0} \operatorname{Id}_{x}+C_{0} \text { strongly in } H^{1}\left(\omega_{0}\right)^{2} \\
& \text { and (up to a subsequence) a.e. in } \omega_{0}, \\
& A_{\delta} \cdot \mathrm{Id}_{x}+a_{\delta} \rightarrow A_{0} \cdot \mathrm{Id}_{x}+a_{0} \text { strongly in } H^{2}\left(\omega_{0}\right) \\
& \text { and (up to a subsequence) a.e. in } \omega_{0} .
\end{aligned}
$$

The strong convergences $\nabla_{x}\left(B_{\delta} \operatorname{Id}_{x}+C_{\delta}\right) \rightarrow \nabla_{x}\left(B_{0} \operatorname{Id}_{x}+C_{0}\right)$ in $L^{2}\left(\omega_{0}\right)^{2 \times 2}$ and $\nabla_{x}\left(A_{\delta} \cdot \operatorname{Id}_{x}+a_{\delta}\right) \rightarrow \nabla_{x}\left(A_{0} \cdot \operatorname{Id}_{x}+a_{0}\right)$ in $H^{1}\left(\omega_{0}\right)^{2}$ mean in particular $B_{\delta} \rightarrow B_{0}$ in $\mathbb{R}^{2 \times 2}$ and $A_{\delta} \rightarrow A_{0}$ in $\mathbb{R}^{2}$. Hence (51)-(52) imply the remaining convergences $C_{\delta} \rightarrow C_{0}$ in $\mathbb{R}^{2}$ and $a_{\delta} \rightarrow a_{0}$ in $\mathbb{R}$, so we are done.

We intend now to identify the limit of the sequence $\left(W_{\delta}^{i}, w_{\delta}^{i}\right)$ for each $i=1,2$.

Lemma 2.9 Let $\left(\widetilde{W}_{0}, \widetilde{w}_{0}\right)=\left(P_{0}+Q_{0}, p_{0}+q_{0}\right)$, where $P_{0}=\operatorname{Ext}_{1} \circ \operatorname{Tr}_{1, \Omega \backslash \bar{\omega}_{0}}^{-1}\left(\nabla_{x} V\right.$ $\left.\llbracket W_{0} \rrbracket ;(0,0)^{\mathrm{T}}\right), Q_{0}=B_{0} V, p_{0}=\operatorname{Ext}_{2} \circ \operatorname{Tr}_{2, \Omega \backslash \bar{\omega}_{0}}^{-1}\left(\llbracket w_{0} \rrbracket ; 0 ; \llbracket \nabla_{x} w_{0} \rrbracket \cdot\left(\nabla_{x} V+\right.\right.$ $\left.\left.\left(\nabla_{x} V\right)^{\mathrm{T}}\right) v_{0} ; 0\right)$, and $q_{0}=A_{0} \cdot V$. Then, for each $i=1,2$, the sequence $\left(W_{\delta}^{i}, w_{\delta}^{i}\right)$ converges strongly to $\left(\widetilde{W}_{0}, \widetilde{w}_{0}\right)$ in $H_{\partial \Omega}^{1}\left(\Omega_{0}\right)^{2} \times H_{\partial \Omega}^{2}\left(\Omega_{0}\right)$ as $\delta \rightarrow 0$.

Proof We first note that, by construction, $\left(\widetilde{W}_{0}, \widetilde{w}_{0}\right) \in H_{\partial \Omega}^{1}\left(\Omega_{0}\right)^{2} \times H_{\partial \Omega}^{2}\left(\Omega_{0}\right)$; furthermore, $P_{0} \equiv(0,0)^{\mathrm{T}}$ and $p_{0} \equiv 0$ in $\omega_{0}$. Since all the functions $P_{\delta}^{i}$ and $p_{\delta}^{i}$ vanish in $\omega_{0}$, it is enough to investigate their convergence in $\Omega \backslash \bar{\omega}_{0}$.

Thanks to the asymptotic expansion (29), continuity of the lifting operator $\operatorname{Tr}_{1, \Omega \backslash \bar{\omega}_{0}}^{-1}$, and Proposition 2.5, we have $P_{\delta}^{1} \rightarrow P_{0}$ strongly in $H^{1}\left(\Omega \backslash \bar{\omega}_{0}\right)^{2}$. From the asymptotic expansion (28), it follows that $Q_{\delta}^{1} \rightarrow Q_{0}$ strongly in $H^{1}\left(\Omega_{0}\right)^{2}$, and $q_{\delta}^{1} \rightarrow q_{0}$ strongly in $H^{2}\left(\Omega_{0}\right)$. The asymptotic expansion

$$
Z_{\delta}=I-\delta\left(\nabla_{x} V+\left(\nabla_{x} V\right)^{\mathrm{T}}\right)+r_{\delta}^{5}, \quad\left\|r_{\delta}^{5}\right\|_{W^{1, \infty}(\Omega)^{2 \times 2}}=o(\delta),
$$

strong convergences $\alpha_{\delta} \rightarrow 1$ and $\beta_{\delta} \rightarrow 0$ in $C^{0,1}\left(\partial \omega_{0}\right)$, and Proposition 2.5 imply that $\left(\nabla_{x} p_{\delta}^{1}\right)^{+} \cdot v_{0} \rightarrow\left(\nabla_{x} p_{0}\right)^{+} \cdot v_{0}$ strongly in $H^{1 / 2}\left(\partial \omega_{0}\right)$. In view of $p_{\delta}^{1+} \equiv p_{0}^{+}$on $\partial \omega_{0}$ and the continuity of the lifting operator $\operatorname{Tr}_{2, \Omega \backslash \bar{\omega}_{0}}^{-1}$, we conclude that $p_{\delta}^{1} \rightarrow p_{0}$ 
strongly in $H^{2}\left(\Omega \backslash \bar{\omega}_{0}\right)$. Consequently, $\left(W_{\delta}^{1}, w_{\delta}^{1}\right)$ converges strongly to $\left(\widetilde{W}_{0}, \widetilde{w}_{0}\right)$ in $H_{\partial \Omega}^{1}\left(\Omega_{0}\right)^{2} \times H_{\partial \Omega}^{2}\left(\Omega_{0}\right)$, as claimed.

By Lemma 2.7 and the continuity of the trace operators $\operatorname{Tr}_{1, \Omega \backslash \bar{\omega}_{0}}$ and $\operatorname{Tr}_{1, \omega_{0}}$, we find $\llbracket W_{\delta} \rrbracket \rightarrow \llbracket W_{0} \rrbracket$ strongly in $H^{1 / 2}\left(\partial \omega_{0}\right)^{2}$. Therefore, using the asymptotic expansion (31), continuity of the lifting operator $\operatorname{Tr}_{1, \Omega \backslash \bar{\omega}_{0}}^{-1}$, and Proposition 2.5, we deduce that $P_{\delta}^{2} \rightarrow P_{0}$ strongly in $H^{1}\left(\Omega \backslash \bar{\omega}_{0}\right)^{2}$. With the aid of the asymptotic expansion (53) and continuity of the trace operators $\operatorname{Tr}_{2, \Omega \backslash \bar{\omega}_{0}}$ and $\operatorname{Tr}_{2, \omega_{0}}$, we derive $p_{\delta}^{2+} \rightarrow p_{0}^{+}$strongly in $H^{3 / 2}\left(\partial \omega_{0}\right)$ and $\left(\nabla_{x} p_{\delta}^{2}\right)^{+} \cdot v_{0} \rightarrow\left(\nabla_{x} p_{0}\right)^{+} \cdot v_{0}$ strongly in $H^{1 / 2}\left(\partial \omega_{0}\right)$. Employing the continuity of the lifting operator $\operatorname{Tr}_{2, \Omega \backslash \bar{\omega}_{0}}^{-1}$, we infer that $p_{\delta}^{2} \rightarrow p_{0}$ strongly in $H^{2}\left(\Omega \backslash \bar{\omega}_{0}\right)$. Additionally, Corollary 2.8 yields $Q_{\delta}^{2} \rightarrow Q_{0}$ strongly in $H^{1}\left(\Omega_{0}\right)^{2}$ and $q_{\delta}^{2} \rightarrow q_{0}$ strongly in $H^{2}\left(\Omega_{0}\right)$. Thus, the strong convergence $\left(W_{\delta}^{2}, w_{\delta}^{2}\right)$ to $\left(\widetilde{W}_{0}, \widetilde{w}_{0}\right)$ in $H_{\partial \Omega}^{1}\left(\Omega_{0}\right)^{2} \times H_{\partial \Omega}^{2}\left(\Omega_{0}\right)$ is established, and the lemma is entirely proved.

Now we have enough tools at hand to prove Theorem 2.2.

Proof of Theorem 2.2 For the calculation of limit (22), we first transform the potential deformation energy $\mathcal{E}\left(\Omega_{\delta} ; \cdot, \cdot\right)$ to the reference configuration $\Omega_{0}$, which leads to

$$
\begin{aligned}
\mathcal{E}_{\delta}\left(\Omega_{0} ; W, w\right)= & h \int_{\Omega \backslash \bar{\omega}_{0}} \operatorname{det}\left(\nabla_{x} T_{\delta}\right) \mathbb{C} E\left(\left(\nabla_{x} T_{\delta}\right)^{-1} ; W\right): E\left(\left(\nabla_{x} T_{\delta}\right)^{-1} ; W\right) \mathrm{d} x \\
& +\frac{h^{3}}{3} \int_{\Omega \backslash \bar{\omega}_{0}} \operatorname{det}\left(\nabla_{x} T_{\delta}\right) \mathbb{C}\left\{\left(\nabla_{x} T_{\delta}\right)^{-\mathrm{T}}\left(\nabla_{x}^{2} w-M_{\delta} \nabla_{x} w\right)\left(\nabla_{x} T_{\delta}\right)^{-1}\right\} \\
& :\left\{\left(\nabla_{x} T_{\delta}\right)^{-\mathrm{T}}\left(\nabla_{x}^{2} w-M_{\delta} \nabla_{x} w\right)\left(\nabla_{x} T_{\delta}\right)^{-1}\right\} \mathrm{d} x \\
& -\int_{\Omega_{0}} \operatorname{det}\left(\nabla_{x} T_{\delta}\right) F_{\delta} \cdot W \mathrm{~d} x-\int_{\Omega_{0}} \operatorname{det}\left(\nabla_{x} T_{\delta}\right) f_{\delta} w \mathrm{~d} x,
\end{aligned}
$$

(W,w) belonging to $H^{1}\left(\Omega_{0}\right)^{2} \times H^{2}\left(\Omega_{0}\right)$. Utilizing the same machinery as in the proof of Lemma 2.4 yields

$$
\begin{aligned}
\mathcal{E}_{\delta}\left(\Omega_{0} ; W, w\right)= & \mathcal{E}\left(\Omega_{0} ; W, w\right)+\frac{\delta}{2} \int_{\Omega \backslash \bar{\omega}_{0}}\left(A_{p}(V ; W, W)+A_{v}(V ; w, w)\right) \mathrm{d} x \\
& -\delta \int_{\Omega_{0}}\left(\operatorname{div}_{x}(F \otimes V) \cdot W+\operatorname{div}_{x}(f V) w\right) \mathrm{d} x+\int_{\Omega_{0}} R_{5}(\delta, W, w) \mathrm{d} x,
\end{aligned}
$$

where

$$
\begin{aligned}
& \left\|R_{5}(\delta, W, w)\right\|_{L^{1}\left(\Omega_{0}\right)} \leq c_{5}(|\delta|)\left(\|(W, w)\|_{H^{1}\left(\Omega_{0}\right)^{2} \times H^{2}\left(\Omega_{0}\right)}^{2}+\|(W, w)\|_{L^{2}\left(\Omega_{0}\right)^{3}}\right) \\
& \quad 0 \leq c_{5}(|\delta|)=o(\delta)
\end{aligned}
$$


Since $\mathbf{T}_{\delta}$ is a bijection between the sets $K^{\delta}\left(\Omega^{\delta}\right)$ and $K_{\delta}\left(\Omega_{0}\right)$, it follows that

$$
\begin{aligned}
\mathcal{E}_{\delta}\left(\Omega_{0} ; W_{\delta}, w_{\delta}\right) & =\mathcal{E}\left(\Omega_{\delta} ; W^{\delta}, w^{\delta}\right)=\inf _{(W, w) \in K^{\delta}\left(\Omega_{\delta}\right)} \mathcal{E}\left(\Omega_{\delta} ; W, w\right) \\
& =\inf _{(W, w) \in K_{\delta}\left(\Omega_{0}\right)} \mathcal{E}_{\delta}\left(\Omega_{0} ; W, w\right) .
\end{aligned}
$$

Let $\delta>0$. Using $\left(\widehat{W}_{\delta}^{1}, \widehat{w}_{\delta}^{1}\right) \in K_{\delta}\left(\Omega_{0}\right)$ as a competitor in (55), we discover

$$
\begin{aligned}
\frac{\mathcal{E}\left(\Omega_{\delta} ; W^{\delta}, w^{\delta}\right)-\mathcal{E}\left(\Omega_{0} ; W_{0}, w_{0}\right)}{\delta} & =\frac{\mathcal{E}_{\delta}\left(\Omega_{0} ; W_{\delta}, w_{\delta}\right)-\mathcal{E}\left(\Omega_{0} ; W_{0}, w_{0}\right)}{\delta} \\
& \leq \frac{\mathcal{E}_{\delta}\left(\Omega_{0} ; \widehat{W}_{\delta}^{1}, \widehat{w}_{\delta}^{1}\right)-\mathcal{E}\left(\Omega_{0} ; W_{0}, w_{0}\right)}{\delta}
\end{aligned}
$$

which implies

$$
\limsup _{\delta \searrow 0} \frac{\mathcal{E}\left(\Omega_{\delta} ; W^{\delta}, w^{\delta}\right)-\mathcal{E}\left(\Omega_{0} ; W_{0}, w_{0}\right)}{\delta} \leq \limsup _{\delta \searrow 0} \frac{\mathcal{E}_{\delta}\left(\Omega_{0} ; \widehat{W}_{\delta}^{1}, \widehat{w}_{\delta}^{1}\right)-\mathcal{E}\left(\Omega_{0} ; W_{0}, w_{0}\right)}{\delta} .
$$

On the other hand, considering $\left(\widehat{W}_{\delta}^{2}, \widehat{w}_{\delta}^{2}\right) \in K_{0}\left(\Omega_{0}\right)$ as a competitor in (6), we arrive at

$$
\frac{\mathcal{E}_{\delta}\left(\Omega_{0} ; W_{\delta}, w_{\delta}\right)-\mathcal{E}\left(\Omega_{0} ; \widehat{W}_{\delta}^{2}, \widehat{w}_{\delta}^{2}\right)}{\delta} \leq \frac{\mathcal{E}_{\delta}\left(\Omega_{0} ; W_{\delta}, w_{\delta}\right)-\mathcal{E}\left(\Omega_{0} ; W_{0}, w_{0}\right)}{\delta},
$$

so that

$$
\liminf _{\delta \searrow 0} \frac{\mathcal{E}_{\delta}\left(\Omega_{0} ; W_{\delta}, w_{\delta}\right)-\mathcal{E}\left(\Omega_{0} ; \widehat{W}_{\delta}^{2}, \widehat{w}_{\delta}^{2}\right)}{\delta} \leq \liminf _{\delta \searrow 0} \frac{\mathcal{E}\left(\Omega_{\delta} ; W^{\delta}, w^{\delta}\right)-\mathcal{E}\left(\Omega_{0} ; W_{0}, w_{0}\right)}{\delta} .
$$

Invoking the asymptotic expansion of the transformed potential deformation energy (54), we can calculate the limit superior on the right-hand side of (57) and the limit inferior on the left-hand side of (59). Direct computations based on Lemmas 2.7 and 2.9 reveal that they are finite and equal. Therefore,

$$
\begin{aligned}
\lim _{\delta \searrow 0} \frac{\mathcal{E}\left(\Omega_{\delta} ; W^{\delta}, w^{\delta}\right)-\mathcal{E}\left(\Omega_{0} ; W_{0}, w_{0}\right)}{\delta}= & \frac{1}{2} \int_{\Omega \backslash \bar{\omega}_{0}}\left(A_{p}\left(V ; W_{0}, W_{0}\right)+A_{v}\left(V ; w_{0}, w_{0}\right)\right) \mathrm{d} x \\
& -\int_{\Omega \backslash \bar{\omega}_{0}}\left(\operatorname{div}_{x}(F \otimes V) \cdot W+\operatorname{div}_{x}(f V) w\right) \mathrm{d} x \\
& +\int_{\Omega \backslash \bar{\omega}_{0}} \sigma\left(W_{0}\right): \varepsilon\left(\widetilde{W}_{0}\right) \mathrm{d} x-\int_{\Omega \backslash \bar{\omega}_{0}} m\left(w_{0}\right): \nabla_{x}^{2} \widetilde{w}_{0} \mathrm{~d} x \\
& -\int_{\Omega_{0}}\left(F \cdot \widetilde{W}_{0}+f \widetilde{w}_{0}\right) \mathrm{d} x .
\end{aligned}
$$


For $\delta<0$, we just have to flip inequality signs in (56) and (58) and reason as before to prove that

$$
\lim _{\delta \nearrow 0} \frac{\mathcal{E}\left(\Omega_{\delta} ; W^{\delta}, w^{\delta}\right)-\mathcal{E}\left(\Omega_{0} ; W_{0}, w_{0}\right)}{\delta}=\lim _{\delta \searrow 0} \frac{\mathcal{E}\left(\Omega_{\delta} ; W^{\delta}, w^{\delta}\right)-\mathcal{E}\left(\Omega_{0} ; W_{0}, w_{0}\right)}{\delta}
$$

In view of the above discussion, the limit in (22) exists and coincides with the righthand side of (60). Thus it remains to show that the right-hand side of (60) is equal to $\mathfrak{G}\left(\Omega_{0} ; V\right)$. To do so, let us set

$$
\begin{aligned}
\int_{\Omega \backslash \bar{\omega}_{0}} \sigma\left(W_{0}\right): \varepsilon\left(\widetilde{W}_{0}\right) \mathrm{d} x & -\int_{\Omega \backslash \bar{\omega}_{0}} m\left(w_{0}\right): \nabla_{x}^{2} \widetilde{w}_{0} \mathrm{~d} x \\
& -\int_{\Omega_{0}}\left(F \cdot \widetilde{W}_{0}+f \widetilde{w}_{0}\right) \mathrm{d} x=I_{p}\left(W_{0}, \widetilde{W}_{0}\right)+I_{v}\left(w_{0}, \widetilde{w}_{0}\right) .
\end{aligned}
$$

An application of the Green formula [17, Theorem 1.17] gives

$$
\begin{aligned}
I_{p}\left(W_{0}, \widetilde{W}_{0}\right)= & \int_{\Omega \backslash \bar{\omega}_{0}}\left(\operatorname{div}_{x} \sigma\left(W_{0}\right)-F\right) \cdot \widetilde{W}_{0} \mathrm{~d} x-\int_{\omega_{0}} F \cdot \widetilde{W}_{0} \mathrm{~d} x \\
& -\left\langle\sigma^{+}\left(W_{0}\right) \nu_{0}, Q_{0}\right\rangle_{1 / 2, \partial \omega_{0}} \\
& -\left\langle\sigma_{\nu_{0}}^{+}\left(W_{0}\right), P_{0}^{+} \cdot v_{0}\right\rangle_{1 / 2, \partial \omega_{0}}-\left\langle\sigma_{\tau_{0}}^{+}\left(W_{0}\right), P_{0 \tau_{0}}^{+}\right\rangle_{1 / 2, \partial \omega_{0}},
\end{aligned}
$$

where $P_{0 \tau_{0}}^{+}=P_{0}^{+}-\left(P_{0}^{+} \cdot v_{0}\right) \nu_{0}$. Since $P_{0}^{+}=(0,0)^{\mathrm{T}}$ on $\partial \omega_{0} \backslash \gamma_{0}$, we find

$$
\begin{aligned}
& \left\langle\sigma_{\nu_{0}}^{+}\left(W_{0}\right), P_{0}^{+} \cdot v_{0}\right\rangle_{1 / 2, \partial \omega_{0}}+\left\langle\sigma_{\tau_{0}}^{+}\left(W_{0}\right), P_{0 \tau_{0}}^{+}\right\rangle_{1 / 2, \partial \omega_{0}}=\left\langle\sigma_{\nu_{0}}^{+}\left(W_{0}\right), P_{0}^{+} \cdot v_{0}\right\rangle_{1 / 2, \gamma_{0}}^{00} \\
& \quad+\left\langle\sigma_{\tau_{0}}^{+}\left(W_{0}\right), P_{0 \tau_{0}}^{+}\right\rangle_{1 / 2, \gamma_{0}}^{00} .
\end{aligned}
$$

Owing to the equilibrium equations (8) and Remark 1.1, we infer

$$
\begin{aligned}
I_{p}\left(W_{0}, \widetilde{W}_{0}\right)= & -\int_{\omega_{0}} F \cdot B_{0} V \mathrm{~d} x-\left\langle\sigma^{+}\left(W_{0}\right) \nu_{0}, B_{0} V\right\rangle_{1 / 2, \partial \omega_{0}} \\
& -\left\langle\sigma_{\nu_{0}}^{+}\left(W_{0}\right), \nabla_{x} V \llbracket W_{0} \rrbracket \cdot v_{0}\right\rangle_{1 / 2, \gamma_{0}}^{00} .
\end{aligned}
$$

Let us turn now to $I_{v}\left(w_{0}, \widetilde{w}_{0}\right)$. We employ the Green formula [17, Theorem 1.19] to deduce

$I_{v}\left(w_{0}, \widetilde{w}_{0}\right)=\int_{\Omega \backslash \bar{\omega}_{0}}\left(-\operatorname{div}_{x} \operatorname{div}_{x} m\left(w_{0}\right)-f\right) \widetilde{w}_{0} \mathrm{~d} x-\int_{\omega_{0}} f \widetilde{w}_{0} \mathrm{~d} x+\left\langle t_{\nu_{0}}^{+}\left(w_{0}\right), q_{0}\right\rangle_{3 / 2, \partial \omega_{0}}$ 


$$
\begin{aligned}
& -\left\langle m_{v_{0}}^{+}\left(w_{0}\right), \nabla_{x} q_{0} \cdot v_{0}\right\rangle_{1 / 2, \partial \omega_{0}}+\left\langle t_{\nu_{0}}^{+}\left(w_{0}\right), p_{0}^{+}\right\rangle_{3 / 2, \partial \omega_{0}} \\
& -\left\langle m_{v_{0}}^{+}\left(w_{0}\right),\left(\nabla_{x} p_{0}\right)^{+} \cdot v_{0}\right\rangle_{1 / 2, \partial \omega_{0}} .
\end{aligned}
$$

Taking into account that $p_{0}^{+}=0$ and $\left(\nabla_{x} p_{0}\right)^{+} \cdot v_{0}=0$ on $\partial \omega_{0} \backslash \gamma_{0}$, we thereby obtain

$$
\begin{aligned}
& \left\langle t_{v_{0}}^{+}\left(w_{0}\right), p_{0}^{+}\right\rangle_{3 / 2, \partial \omega_{0}}-\left\langle m_{\nu_{0}}^{+}\left(w_{0}\right),\left(\nabla_{x} p_{0}\right)^{+} \cdot v_{0}\right\rangle_{1 / 2, \partial \omega_{0}} \\
& =\left\langle t_{\nu_{0}}^{+}\left(w_{0}\right), p_{0}^{+}\right\rangle_{3 / 2, \gamma_{0}}^{00}-\left\langle m_{v_{0}}^{+}\left(w_{0}\right),\left(\nabla_{x} p_{0}\right)^{+} \cdot v_{0}\right\rangle_{1 / 2, \gamma_{0}}^{00} .
\end{aligned}
$$

Furthermore, the equilibrium equation (10) and Remark 1.1 yield

$$
\begin{aligned}
I_{v}\left(w_{0}, \widetilde{w}_{0}\right)= & -\int_{\omega_{0}} f A_{0} \cdot V \mathrm{~d} x+\left\langle t_{\nu_{0}}^{+}\left(w_{0}\right), A_{0} \cdot V\right\rangle_{3 / 2, \partial \omega_{0}} \\
& -\left\langle m_{\nu_{0}}^{+}\left(w_{0}\right), \nabla_{x}\left(A_{0} \cdot V\right) \cdot v_{0}\right\rangle_{1 / 2, \partial \omega_{0}} \\
& -\left\langle m_{\nu_{0}}^{+}\left(w_{0}\right), \llbracket \nabla_{x} w_{0} \rrbracket \cdot\left(\nabla_{x} V+\left(\nabla_{x} V\right)^{\mathrm{T}}\right) \nu_{0}\right\rangle_{1 / 2, \gamma_{0}}^{00} .
\end{aligned}
$$

Combining (60)-(62) completes the proof.

Remark 2.10 The structural assumption (1) arises in dimension reductions from 3D to 2D within the Kirchhoff-Love energy scaling regime [8-10]. In the case of an undamaged elastic plate, this assumption provides in particular that the limit stored elastic energy is a sum of uncoupled bending and stretching energies.

From a mathematical standpoint, we have no difficulty in treating a more general case in which the elastic properties of the plate depend on the spartial variable $x$ as in [17]. Indeed, let $\mathbb{C}^{p}, \mathbb{C}^{v} \in C^{1}(\bar{\Omega})^{2 \times 2 \times 2 \times 2}$ be fourth-order tensors. For each $n=p, v$, the tensor $\mathbb{C}^{n}$ is assumed to be positive, i.e., there exists $c_{\mathbb{C}^{n}}>0$ such that

$$
\mathbb{C}^{n}(x) X: X \geq c_{\mathbb{C}^{n}}|X|^{2} \text { for all } X \in \mathbb{R}_{\text {sym }}^{2 \times 2} \text { and all } x \in \Omega,
$$

and symmetric

$$
\mathbb{C}_{i j k l}^{n}=\mathbb{C}_{i j l k}^{n}=\mathbb{C}_{k l i j}^{n}, \quad i, j, k, l=1,2
$$

We denote by $\mathbb{C}^{n, V}$ the fourth-order tensor with components $\mathbb{C}_{i j k l}^{n, V}=\nabla_{x} \mathbb{C}_{i j k l}^{n} \cdot V$, $i, j, k, l=1,2$, and replace the constitutive relations (2),(3) (see also (9),(11)) by

$$
\sigma=\mathbb{C}^{p} \varepsilon(W), \quad m=-\mathbb{C}^{v} \nabla_{x}^{2} w
$$

and the bilinear forms (23),(24) by

$$
\begin{array}{r}
A_{p}(V ; U, W)=\operatorname{div}_{x} V \sigma(U): \varepsilon(W)-\sigma(U): E\left(\nabla_{x} V ; W\right) \\
-\sigma(W): E\left(\nabla_{x} V ; U\right)+\mathbb{C}^{p, V} \varepsilon(U): \varepsilon(W),
\end{array}
$$




$$
\begin{aligned}
A_{v}(V ; u, w)=- & \operatorname{div}_{x} V m(u): \nabla_{x}^{2} w+m(u):\left(2 \nabla_{x}^{2} w \nabla_{x} V+N \nabla_{x} w\right) \\
& +m(w):\left(2 \nabla_{x}^{2} u \nabla_{x} V+N \nabla_{x} u\right)+\mathbb{C}^{v, V} \nabla_{x}^{2} u: \nabla_{x}^{2} w .
\end{aligned}
$$

Exploiting the asymptotic representations

$$
\mathbb{C}^{n} \circ T_{\delta}=\mathbb{C}^{n}+\delta \mathbb{C}^{n, V}+r_{\delta}^{n 6}, \quad\left\|r_{\delta}^{n 6}\right\|_{L^{\infty}(\Omega)^{2 \times 2 \times 2 \times 2}}=o(\delta),
$$

we can easily adapt the arguments above to show that all our results remain valid.

Remark 2.11 (Griffith formula) We now demonstrate how one can apply the result obtained to derive the Griffith formula for the energy release rate associated with extension of the crack along the interface.

We assume that there exists a simple curve $\Lambda$ of class $C^{2,1}$ such that $\gamma_{0} \varsubsetneqq \Lambda \subset \partial \omega_{0}$, and let $\lambda:\left(0, \mathcal{H}^{1}(\Lambda)\right) \rightarrow \mathbb{R}^{2}$ be its arc-length parametrization, whereas $\gamma_{0}=\lambda(0, l)$ with $l=\mathcal{H}^{1}\left(\gamma_{0}\right)$. For technical reason, we require the validity of $\mathbf{G 1}$ for the domain $\Omega \backslash \bar{\Lambda}$. The cylindrical surface $\Lambda^{3 \mathrm{D}}$ is a prescribed crack path. For $\delta \in\left(-l, \mathcal{H}^{1}\left(\Lambda \backslash \gamma_{0}\right)\right)$, the admissible crack set is $\gamma_{\delta}^{3 \mathrm{D}}=\lambda(0, l+\delta) \times(-h, h)$, and we put $\Omega_{\delta}=\Omega \backslash \bar{\gamma}_{\delta}$.

The energy release rate associated with crack extension $\mathcal{E} \mathcal{R} \mathcal{R}\left(\Omega_{0}\right)$ is formally defined as the opposite of the derivative of the potential deformation energy with respect to the crack length:

$$
\mathcal{E R R}\left(\Omega_{0}\right)=-\lim _{\delta \rightarrow 0} \frac{\mathcal{E}\left(\Omega_{\delta} ; W^{\delta}, w^{\delta}\right)-\mathcal{E}\left(\Omega_{0} ; W_{0}, w_{0}\right)}{\delta},
$$

where $\left(W^{\delta}, w^{\delta}\right)$ and $\left(W_{0}, w_{0}\right)$ are the displacement fields corresponding to $\Omega_{\delta}, \delta \neq 0$, and $\Omega_{0}$, respectively. Thanks to the regularity assumptions on $\Lambda$, we can find $\mu>0$ and $\psi_{l} \in C^{2,1}\left(\left[\lambda_{1}(l)-\mu, \lambda_{1}(l)+\mu\right]\right)$ such that

$$
\Lambda=\left\{\left(x_{1}, \psi_{l}\left(x_{1}\right)\right): x_{1} \in\left[\lambda_{1}(l)-\mu, \lambda_{1}(l)+\mu\right]\right\} .
$$

Choose a cut-off function $\theta \in C^{\infty}\left(\mathbb{R}^{2}\right)$ so that $\theta=1$ on $B_{\mu / 3}(0)$ and $\theta=0$ outside $B_{\mu / 2}(0)$, and define the mapping $T_{l, \delta}: \mathbb{R}^{2} \rightarrow \mathbb{R}^{2}$ by

$$
T_{l, \delta}(x)=x+\left(\begin{array}{c}
\left(\lambda_{1}(l+\delta)-\lambda_{1}(l)\right) \theta(\lambda(l)-x) \\
\psi_{l}\left(x_{1}+\left(\lambda_{1}(l+\delta)-\lambda_{1}(l)\right) \theta(\lambda(l)-x)\right)-\psi_{l}\left(x_{1}\right)
\end{array}\right) .
$$

Hence there exists $\delta_{0}>0$ such that $T_{l, .} \in C^{2,1}\left(\left[-\delta_{0}, \delta_{0}\right] \times \mathbb{R}^{2}\right)^{2}$ and, for every $\delta \in\left[-\delta_{0}, \delta_{0}\right]$, the mapping $T_{l, \delta}$ is a $C^{2,1}$-diffeomorphism on $\mathbb{R}^{2}$ with $T_{l, \delta}\left(\Omega_{0}\right)=\Omega_{\delta}$, $T_{l, \delta}(\lambda(l))=\lambda(l+\delta), T_{l, \delta}\left(\gamma_{0}\right)=\gamma_{\delta}$, and $T_{l, \delta}\left(\omega_{0}\right)=\omega_{0}$, see [24, Lemma 3.5]. By construction, the vector field

$$
V_{\mathrm{Gr}}(x)=\left.\partial_{\delta}\left(T_{l, \delta}(x)\right)\right|_{\delta=0}=\lambda_{1}^{\prime}(l) \theta(\lambda(l)-x)\left(1, \psi_{l, 1}\left(x_{1}\right)\right)^{\mathrm{T}}
$$

belongs to $C^{1,1}\left(\mathbb{R}^{2}\right)^{2}$ and is compactly supported in $\Omega$. We thus deduce that the energy release rate associated with crack extension (63) is well defined and can be calculated as 


$$
\operatorname{ERR}\left(\Omega_{0}\right)=-\mathfrak{G}\left(\Omega_{0} ; V_{\mathrm{Gr}}\right)
$$

We conclude this remark and paper by noting that, in view of the definition (63), the energy release rate associated with crack extension $\mathcal{E} \mathcal{R} \mathcal{R}\left(\Omega_{0}\right)$ is independent of the choice of the cut-off function $\theta$.

Acknowledgements E. Rudoy acknowledges the partial financial support by the Russian Foundation for Basic Research under Grant 18-29-10007. V. Shcherbakov is supported by the Alexander von Humboldt Foundation.

Funding Open Access funding enabled and organized by Projekt DEAL.

Open Access This article is licensed under a Creative Commons Attribution 4.0 International License, which permits use, sharing, adaptation, distribution and reproduction in any medium or format, as long as you give appropriate credit to the original author(s) and the source, provide a link to the Creative Commons licence, and indicate if changes were made. The images or other third party material in this article are included in the article's Creative Commons licence, unless indicated otherwise in a credit line to the material. If material is not included in the article's Creative Commons licence and your intended use is not permitted by statutory regulation or exceeds the permitted use, you will need to obtain permission directly from the copyright holder. To view a copy of this licence, visit http://creativecommons.org/licenses/by/4.0/.

\section{References}

1. Almi, S.: Energy release rate and quasi-static evolution via vanishing viscosity in a fracture model depending on the crack opening. ESAIM: COCV 23, 791-826 (2017)

2. Anicic, S., Dret, H.L., Raoult, A.: The infinitesimal rigid displacement lemma in Lipschitz coordinates and application to shells with minimal regularity. Math. Meth. Appl. Sci. 27, 1283-1299 (2004)

3. Bilotta, A., Morassi, A., Rosset, E., Turco, E., Vessella, S.: Numerical size estimates of inclusions in Kirchhoff-Love elastic plates. Int. J. Solids Struct. 168, 58-72 (2019)

4. Budiansky, B., Rice, J.: Conservation laws and energy-release rates. J. Appl. Mech. 40, 201-203 (1973)

5. Chen, Y.H.: Advances in Conservation Laws and Energy Release Rates: Theoretical Treatments and Applications. Springer, Dordrecht (2013)

6. Ciarlet, P.G.: Mathematical Elasticity. Three-Dimensioned Elasticity, vol. 1. North-Holland, Amsterdam (1988)

7. Delfour, M.C.: Control, shape, and topological derivatives via minimax differentiability of Lagrangians. In: Falcone, M., Ferretti, R., Grüne, L., McEneaney, W. (eds.) Numerical Methods for Optimal Control Problems. Springer INdAM Series, vol. 29, pp. 137-164. Springer, Berlin (2018)

8. Freddi, L., Paroni, R., Zanini, C.: Dimension reduction of a crack evolution problem in a linearly elastic plate. Asymptot. Anal. 70, 101-123 (2010)

9. Freddi, L., Paroni, R., Roubíček, T., Zanini, C.: Quasistatic delamination models for Kirchhoff-Love plates. Z. Angew. Math. Mech. 91, 845-865 (2011)

10. Freddi, L., Roubíček, T., Zanini, C.: Quasistatic delamination of sandwich-like Kirchhoff-Love plates. J. Elasticity 113, 219-250 (2013)

11. Geymonat, G.: Trace theorems for Sobolev spaces on Lipschitz domains. Necessary conditions. Ann. Math. Blaise Pascal 14, 187-197 (2007)

12. Griffith, A.A.: The phenomena of rupture and flow in solids. Philos. Trans. R. Soc. Lond. Ser. A. 221, 163-198 (1921)

13. Gonzalez Granada, J.R., Gwinner, J., Kovtunenko, V.A.: On the shape differentiability of objectives: a Lagrangian approach and the Brinkman problem. Axioms 7, 76 (2018)

14. Grisvard, P.: Elliptic Problems in Nonsmooth Domains. Pitman, Boston (1985)

15. Hintermüller, M., Kovtunenko, V.A.: From shape variation to topological changes in constrained minimization: a velocity method-based concept. Optim. Method. Soft. 26, 513-532 (2011)

16. Khludnev, A.M.: On extremal shapes of cuts in a plate. Izv. Ross. Akad Nauk. MTT 1, 170-176 (1992) 
17. Khludnev, A.M.: Problem of a crack on the boundary of a rigid inclusion in an elastic plate. Mech. Solids 45, 733-742 (2010)

18. Khludnev, A.M.: Elasticity Problems in Nonsmooth Domains. Fizmatlit, Moscow (2010). [in Russian]

19. Khludnev, A.M., Kovtunenko, V.A.: Analysis of Cracks in Solids. WIT Press, Boston (2000)

20. Khludnev, A.M., Shcherbakov, V.V.: A note on crack propagation paths inside elastic bodies. Appl. Math. Lett. 79, 80-84 (2018)

21. Khludnev, A.M., Kovtunenko, V.A., Tani, A.: Evolution of a crack with kink and non-penetration. J. Math. Soc. Jpn. 60, 1219-1253 (2008)

22. Khludnev, A.M., Novotny, A.A., Sokołowski, J., Zochowski, A.: Shape and topology sensitivity analysis for cracks in elastic bodies on boundaries of rigid inclusions. J. Mech. Phys. Solids 57, 1718-1732 (2009)

23. Khludnev, A.M., Leugering, G., Specovius-Neugebauer, M.: Optimal control of inclusion and crack shapes in elastic bodies. J. Optim. Theory Appl. 155, 54-78 (2012)

24. Knees, D., Mielke, A., Zanini, C.: On the inviscid limit of a model for crack propagation. Math. Models Methods Appl. Sci. 18, 1529-1569 (2008)

25. Kovtunenko, V.A.: Primal-dual methods of shape sensitivity analysis for curvilinear cracks with nonpenetration. IMA J. Appl. Math. 71, 635-657 (2006)

26. Kovtunenko, V.A., Kunisch, K.: Problem of crack perturbation based on level sets and velocities. Z. Angew. Math. Mech. 87, 809-830 (2007)

27. Kovtunenko, V.A., Leugering, G.: A shape-topological control problem for nonlinear crack-defect interaction: The antiplane variational model. SIAM J. Control Optim. 54, 1329-1351 (2016)

28. Kovtunenko, V.A., Ohtsuka, K.: Shape differentiability of Lagrangians and application to Stokes problem. SIAM J. Control Optim. 56, 3668-3684 (2018)

29. Lazarev, N.P.: Shape sensitivity analysis of the energy integrals for the Timoshenko-type plate containing a crack on the boundary of a rigid inclusion. Z. Angew. Math. Phys. 66, 2025-2040 (2015)

30. Lazarev, N.P.: Existence of an optimal size of a delaminated rigid inclusion embedded in the KirchhoffLove plate. Bound. Value Probl. 2015, 180 (2015)

31. Lazarev, N.P., Itou, H.: Optimal location of a rigid inclusion in equilibrium problems for inhomogeneous Kirchhoff-Love plates with a crack. Math. Mech. Solids 24, 3743-3752 (2019)

32. Lazarev, N.P., Rudoy, E.M.: Shape sensitivity analysis of Timoshenko's plate with a crack under the nonpenetration condition. Z. Angew. Math. Mech. 94, 730-739 (2014)

33. Leugering, G., Sokołowski, J., Zochowski, A.: Control of crack propagation by shape-topological optimization. Discrete Cont. Dyn A 35, 2625-2657 (2015)

34. Mielke, A., Roubíček, T.: Rate-Independent Systems: Theory and Application. Applied Mathematical Sciences, vol. 193. Springer, New York (2015)

35. Mielke, A., Roubíček, T., Stefanelli, U.: $\Gamma$-limits and relaxations for rate-independent evolutionary problems. Calc. Var. PDEs. 31, 387-416 (2008)

36. Morassi, A., Rosset, E., Vessella, S.: Stable determination of a rigid inclusion in an anisotropic elastic plate. SIAM J. Math. Anal. 44, 2204-2235 (2012)

37. Morassi, A., Rosset, E., Vessella, S.: Optimal stability in the identification of a rigid inclusion in an isotropic Kirchhoff-Love plate. SIAM J. Math. Anal. 51, 731-747 (2019)

38. Necas, J.: Direct Methods in the Theory of Elliptic Equations. Springer, Berlin (2012)

39. Plotnikov, P.I., Rudoy, E.M.: Shape sensitivity analysis of energy integrals for bodies with rigid inclusions and cracks. Dokl. Math. 84, 681-684 (2012)

40. Rudoy, E.M.: Differentiation of energy functionals in two-dimensional elasticity theory for solids with curvilinear cracks. J. Appl. Mech. Technol. Phys. 45, 843-852 (2004)

41. Rudoy, E.M.: Differentiation of energy functionals in the problem on a curvilinear crack with possible contact between the shores. Mech. Solids 42, 935-946 (2007)

42. Rudoy, E.M.: Asymptotics of the energy functional for a fourth-order mixed boundary value problem in a domain with cut. Sib. Math. J. 50, 341-354 (2009)

43. Rudoy, E.M.: Shape derivative of the energy functional in a problem for a thin rigid inclusion in an elastic body. Z. Angew. Math. Phys. 66, 1923-1937 (2015)

44. Shcherbakov, V.: Shape optimization of rigid inclusions in elastic plates with cracks. Z. Angew. Math. Phys. 67, 71 (2016)

45. Shcherbakov, V.: Energy release rates for interfacial cracks in elastic bodies with thin semirigid inclusions. Z. Angew. Math. Phys. 68, 26 (2017) 
46. Sokolowski, J., Zolesio, J.-P.: Introduction to Shape Optimization. Shape Sensitivity Analysis. Springer, Berlin (1992)

47. Sturm, K.: Minimax Lagrangian approach to the differentiability of non-linear PDE constrained shape functions without saddle point assumption. SIAM J. Control Optim. 53, 2017-2039 (2015)

48. Temam, R.: Mathematical Problems in Plasticity. Gauthier-Villars, Paris (1985)

49. Xavier, M., Novotny, A.A., Sokołowski, J.: Crack growth control based on the topological derivative of the Rice's integral. J. Elasticity 134, 175-191 (2019)

Publisher's Note Springer Nature remains neutral with regard to jurisdictional claims in published maps and institutional affiliations. 